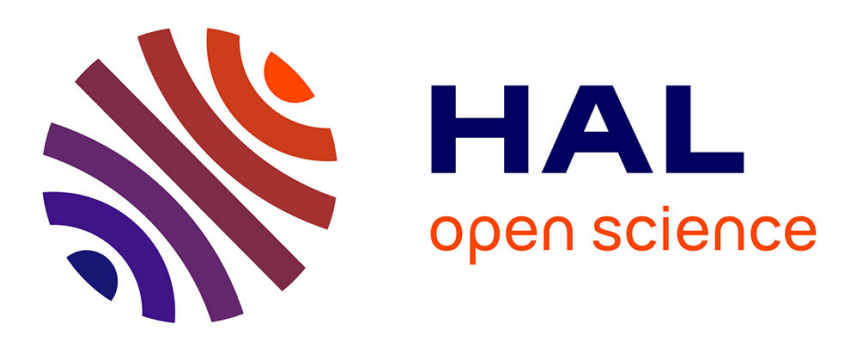

\title{
Global weak solutions to the 1D compressible Euler equations with radiation
}

\author{
Xavier Blanc, B. Ducomet
}

\section{To cite this version:}

Xavier Blanc, B. Ducomet. Global weak solutions to the 1D compressible Euler equations with radiation. Communications in Mathematical Sciences, 2015, 13 (7), pp.1875-1904. 10.4310/CMS.2015.v13.n7.a10 . hal-01143249

\section{HAL Id: hal-01143249 \\ https://hal.science/hal-01143249}

Submitted on 17 Apr 2015

HAL is a multi-disciplinary open access archive for the deposit and dissemination of scientific research documents, whether they are published or not. The documents may come from teaching and research institutions in France or abroad, or from public or private research centers.
L'archive ouverte pluridisciplinaire HAL, est destinée au dépôt et à la diffusion de documents scientifiques de niveau recherche, publiés ou non, émanant des établissements d'enseignement et de recherche français ou étrangers, des laboratoires publics ou privés. 


\author{
Xavier Blanc, Bernard Ducomet
}

\title{
Global weak solutions to the 1D compressible Euler equations with radiation
}

\begin{abstract}
SUMMARY
We consider the Cauchy problem for the equations of one-dimensional motion of a compressible inviscid gas coupled with radiation through a radiative transfer equation. Assuming suitable hypotheses on the transport coefficients and the data, we prove that the problem admits a weak solution. More precisely, we show that a sequence of approximate solutions constructed by a generalized Glimm's scheme admits a subsequence converging to an entropic solution of the problem.
\end{abstract}

Keywords: compressible, Euler, one-dimensional symmetry, radiative transfer. AMS subject classification: $35 \mathrm{Q} 30,76 \mathrm{~N} 10$

\section{Introduction}

The purpose of radiation hydrodynamics is to include the effects of radiation into the hydrodynamical framework. When the equilibrium holds between the matter and the radiation, a simple way to do that is to include local radiative terms into the state functions and the transport coefficients. On the other hand, radiation is described by photons, which are massless particles traveling at the speed $c$ of light, characterized by their frequency $\nu \in \mathbb{R}_{+}$, their energy $E=h \nu$ (where $h$ is the Planck's constant), their momentum $\vec{p}=\frac{\hbar \nu}{c} \vec{\Omega}$, where $\vec{\Omega} \in S^{2}$ is a vector of the 2-unit sphere. Statistical mechanics allows us to describe macroscopically an assembly of massless photons of energy $E$ and momentum $\vec{p}$ by using a distribution function: the radiative intensity $I(x, t, \vec{\Omega}, \nu)$. Using this intensity, one can derive global quantities by integrating with respect to the angular and frequency variables: the spectral radiative energy density $E_{R}(x, t)$ per unit volume is then $E_{R}(x, t):=\frac{1}{c} \iint I(x, t, \vec{\Omega}, \nu) d \Omega d \nu$, and the spectral radiative flux $\vec{F}_{R}(x, t)=\iint \vec{\Omega} I(x, t, \vec{\Omega}, \nu) d \Omega d \nu$.

In the absence of radiation the hydrodynamical system is derived from the fundamental conservation laws (mass, momentum and energy) by using the Boltzmann's equation satisfied by the $f_{m}(x, \vec{v}, t)$ and ChapmanEnskog's expansion. One gets then formally the compressible Euler system for matter. When radiation is taken into account at a macroscopic level, supplementary source terms appear, coupling matter variables to radiative intensity $I$, which is supposed to satisfy a transport equation: the so called radiative transfer equation, an integro-differential equation early discussed by Chandrasekhar in [4].

Supposing that the matter is at local thermodynamical equilibrium (LTE) and in the non-relativistic framework (the velocity of matter is much less than the velocity of light: $\vec{v}^{2} \ll c^{2}$ ), the coupled system satisfied by the density $\rho$, the velocity $\vec{u}$, the temperature $\vartheta$ and the radiative intensity $I$ in $\mathbb{R}^{3}$ reads $[3][26][27]$

$$
\left\{\begin{array}{l}
\partial_{t} \rho+\operatorname{div}_{x}(\rho \vec{v})=0, \\
\partial_{t}(\rho \vec{v})+\operatorname{div}_{x}(\rho \vec{v} \otimes \vec{v})+\nabla_{x} p=-\overrightarrow{\mathcal{S}_{F}}, \\
\partial_{t}(\rho E)+\operatorname{div}_{x}((\rho E+p) \vec{v})=-\mathcal{S}_{E}, \\
\frac{1}{c} \partial_{t} I+\vec{\Omega} \cdot \nabla_{x} I=\mathcal{S},
\end{array}\right.
$$

where $E=\frac{1}{2} \vec{v}^{2}+e$ is the total energy with $e$ the internal energy, $p$ the pressure, and $\vec{S}_{F}$ and $S_{E}$ are the radiative force and energy source terms, described below.

Let us describe the various coupling terms in the right-hand sides of (1) (see[25]). 
In the radiative source splitted into two parts $\mathcal{S}=S_{a, e}+S_{s}$, the first contribution

$$
S_{a, e}(x, t, \vec{\Omega}, \nu)=-\sigma_{a} I(x, t, \vec{\Omega}, \nu)+\sigma_{a} B(x, t, \nu),
$$

is the absorption-emission contribution, and the second one

$$
S_{s}(x, t, \vec{\Omega}, \nu)=-\sigma_{s} I(x, t, \vec{\Omega}, \nu)+\frac{\sigma_{s}}{\pi} \int_{S^{2}} I\left(x, t, \vec{\Omega}^{\prime}, \nu\right) d \vec{\Omega}^{\prime},
$$

is the scattering contribution.

The radiative energy is

$$
\mathcal{S}_{E}(x, t):=\int_{\mathbb{R}_{+}} \int_{S^{2}} S(x, t, \vec{\Omega}, \nu) d \vec{\Omega} d \nu
$$

The radiative flux is

$$
\overrightarrow{\mathcal{S}}_{F}(x, t):=\frac{1}{c} \int_{\mathbb{R}_{+}} \int_{S^{2}} \vec{\Omega} S(x, t, \vec{\Omega}, \nu) d \vec{\Omega} d \nu .
$$

In the radiative transfer equation (the last equation of (1)) the functions $\sigma_{a}$ and $\sigma_{s}$ appearing in the radiative source $\mathcal{S}$ describe in a phenomenological way the absorption-emission and scattering properties (frequency and angular transitions) of the interaction photon-matter and the function $B$ describes the thermodynamical equilibrium distribution.

Let us note that the foundations of the previous system have been described by Pomraning [27] and Mihalas and Weibel-Mihalas [26] in the full framework of special relativity (oversimplified in the previous considerations). The coupled system (1) has been recently investigated by Lowrie, Morel, Hittinger [25], Buet, Després [3] with a special attention to asymptotic regimes, and by Dubroca-Feugeas [7], Lin [20] and Lin-Coulombel-Goudon [21] for numerical aspects. Concerning the existence of solutions, a proof of local-in-time existence and blow-up of solutions has been proposed by Zhong and Jiang [33] (see also the recent papers by Jiang and Wang [15] [16] for a 1D related "Euler -Boltzmann" model), moreover a simplified version of the system has been investigated by Golse and Perthame [11].

As our goal is to prove global existence of solutions for the system (1), we restrict from now our study to the mono-dimensional geometry. The system (1) rewrites

$$
\left\{\begin{array}{l}
\partial_{t} \rho+\partial_{x}(\rho v)=0 \\
\partial_{t}(\rho v)+\partial_{x}\left(\rho v^{2}+p\right)=-\mathcal{S}_{F} \\
\left.\partial_{t}(\rho E)+\partial_{x}((\rho E+p) v)\right)=-\mathcal{S}_{E} \\
\frac{1}{c} \partial_{t} I+\omega \partial_{x} I=\mathcal{S}
\end{array}\right.
$$

where $E=\frac{1}{2} v^{2}+e$ is the total energy with $e(\rho, S)$ the internal energy and $p(\rho, S)$ the pressure.

In order to simplify the study of the fluid part of the system we can use $\rho, v$ and $S$ as new variables $(\vartheta$ and $S$ being the temperature and the entropy of matter) and using the thermodynamical identity $\vartheta d S=d E+p d\left(\frac{1}{\rho}\right)$, we rewrite (1) as

$$
\left\{\begin{array}{c}
\partial_{t} \rho+\partial_{x}(\rho v)=0 \\
\partial_{t} v+v \partial_{x} v+\frac{1}{\rho} \partial_{x} p=-\frac{1}{\rho} \mathcal{S}_{F} \\
\partial_{t} S+v \partial_{x} S=\frac{1}{\rho \vartheta}\left(v \mathcal{S}_{F}-\mathcal{S}_{E}\right)
\end{array}\right.
$$

and the transfer equation is

$$
\frac{1}{c} \partial_{t} I+\omega \partial_{x} I=\mathcal{S}
$$

for $(x, t) \in \mathbb{R} \times \mathbb{R}_{+}$, where the pressure is $p=p(\rho, S)$. 
It will be convenient to use in the sequel the vector notation $U:=\left(\begin{array}{c}\rho \\ v \\ S\end{array}\right)$.

Then (3) rewrites

$$
\partial_{t} U+f_{U} \partial_{x} U=g
$$

with

$$
f_{U}(U):=\left(\begin{array}{ccc}
v & \rho & 0 \\
\frac{p_{\rho}}{\rho} & v & \frac{p_{S}}{\rho} \\
0 & 0 & v
\end{array}\right),
$$

and

$$
g(I, U):=\left(\begin{array}{c}
0 \\
-\frac{1}{\rho} \mathcal{S}_{F} \\
\frac{1}{\rho \vartheta}\left(v \mathcal{S}_{F}-\mathcal{S}_{E}\right)
\end{array}\right) .
$$

After an elementary computation, putting $c^{2}=p_{\rho}$, one gets the three eigenpairs for $f_{U}(U)$

$$
\begin{array}{ll}
\lambda_{1}(U)=v-c, & r_{1}(U)=\left(\begin{array}{c}
\rho \\
-c \\
0
\end{array}\right), \\
\lambda_{2}(U)=v, & r_{2}(U)=\left(\begin{array}{c}
p_{S} \\
0 \\
-c^{2}
\end{array}\right),
\end{array}
$$

and

$$
\lambda_{3}(U)=v+c, \quad r_{3}(U)=\left(\begin{array}{c}
\rho \\
c \\
0
\end{array}\right) .
$$

The corresponding pairs of Riemann invariants are

$$
\begin{gathered}
Z_{1}^{(1)}=S, \quad Z_{1}^{(2)}=v+\int^{\rho} \frac{c(w, S)}{w} d w, \\
Z_{2}^{(1)}=v, \quad Z_{2}^{(2)}=p(\rho, S),
\end{gathered}
$$

and

$$
Z_{3}^{(1)}=S, \quad Z_{3}^{(2)}=v-\int^{\rho} \frac{c(w, S)}{w} d w .
$$

In (2) the radiative intensity $I=I(x, t, \nu, \omega)$, depends also on two extra variables: the radiation frequency $\nu \in \mathbb{R}_{+}$and the angular variable $\omega \in S^{1}:=[-1,1]$.

The absorption-emission and scattering terms rewrite

$$
\mathcal{S}_{a, e}(x, t, \nu, \omega)=\sigma_{a}(t, \nu, \rho, \vartheta)[B(\nu, \vartheta)-I(x, t, \nu, \omega)],
$$

and

$$
\mathcal{S}_{s}(x, t, \nu, \omega)=\sigma_{s}(t, \nu, \rho, \vartheta)[\tilde{I}(x, t, \nu)-I(x, t, \nu, \omega)],
$$

where $\tilde{I}(x, t, \nu):=\frac{1}{2} \int_{-1}^{1} I(x, t, \nu, \omega) d \omega$. 
The function $B(\nu, \vartheta)$ depending on the temperature and the frequency, describes the equilibrium state

$$
B(\nu, \vartheta)=2 h \nu^{3} c^{-2}\left(e^{\frac{h \nu}{k^{\vartheta}}}-1\right)^{-1}
$$

where $k_{B}$ is the Boltzmann's constant and $h$ is the Planck's constant, and corresponds to the Planck's equilibrium distribution of photons in a cavity at temperature $\vartheta$ (black body).

The coefficients $\sigma_{a}$ and $\sigma_{s}$ are positive but their evaluation is a difficult problem of quantum mechanics and their general form is not known (an expression of $\sigma_{a}$ used for stars of moderate mass is given by the Kramers formula $\sigma_{a}(\nu, \vartheta)=\frac{C(\vartheta)}{\nu^{3}}\left(1-e^{-\frac{h \nu}{k_{B} \vartheta}}\right)$, where $C$ is a positive function).

In the following we will also assume that $\sigma_{a}$ and $\sigma_{s}$ are positive, and bounded from above: there exists $\overline{\sigma_{a}}(t), \overline{\sigma_{s}}(t)>0$ in $L^{1}\left(\mathbb{R}^{+}\right) \cap C^{0}\left(\mathbb{R}^{+}\right)$(that is, integrable and continuous) such that

$$
0 \leqslant \sigma_{a}(t, \nu, \rho, \vartheta) \leqslant \overline{\sigma_{a}}(t)
$$

and

$$
0 \leqslant \sigma_{s}(t, \nu, \rho, \vartheta) \leqslant \overline{\sigma_{s}}(t)
$$

Remark 1. The above assumptions are very restrictive. In particular, they do not allow $\sigma_{a}$ and $\sigma_{s}$ to depend only on $\rho, \vartheta$ and $\nu$, as it is physically relevant. The meaning of this, which is made precise below in (21), (22), (23), (24), is that the coupling between radiation and hydrodynamics should be weak in the limit $t \rightarrow \infty$. This limitation is not satisfactory, and is closely linked with the method we use here to prove our result.

We define the radiative energy

$$
E_{R}:=\frac{1}{c} \int_{-1}^{1} \int_{0}^{\infty} I(x, t, \nu, \omega) d \nu d \omega
$$

the radiative flux

$$
F_{R}:=\int_{-1}^{1} \int_{0}^{\infty} \omega I(x, t, \nu, \omega) d \nu d \omega
$$

and the radiative pressure

$$
P_{R}:=\frac{1}{c} \int_{-1}^{1} \int_{0}^{\infty} \omega^{2} I(x, t, \nu, \omega) d \nu d \omega,
$$

Finally, the radiative energy source rewrites

$$
\mathcal{S}_{E}:=\int_{-1}^{1} \int_{0}^{\infty} \mathcal{S}(x, t, \nu, \omega) d \nu d \omega
$$

and the radiative force

$$
\mathcal{S}_{F}:=\frac{1}{c} \int_{-1}^{1} \int_{0}^{\infty} \omega \mathcal{S}(x, t, \nu, \omega) d \nu d \omega .
$$

From the equation (4) and the definitions (9)-(13), one also derives, after integrating in frequency and angular variables, the equations

$$
\left\{\begin{array}{c}
\partial_{t} E_{R}+\partial_{x} F_{R}=\mathcal{S}_{E} \\
\frac{1}{c^{2}} \partial_{t} F_{R}+\partial_{x} P_{R}=\mathcal{S}_{F}
\end{array}\right.
$$

We consider finally the Cauchy problem

$$
\left\{\begin{aligned}
\partial_{t} U(x, t)+\partial_{x}(f(U(x, t))) & =g(U(x, t), x, t) \\
\frac{1}{c} \partial_{t} I(x, t, \nu, \omega)+\omega \partial_{x} I(x, t, \nu, \omega) & =\mathcal{S}(U(x, t), I(x, t, \nu, \omega)),
\end{aligned}\right.
$$

with initial conditions

$$
\left.U\right|_{t=0}=U_{0}(x)=\mid \begin{gathered}
U_{\infty} \text { for } x<-N \\
U^{0}(x) \text { for }|x| \leqslant N \\
U_{\infty} \text { for } x>N
\end{gathered}
$$


and for any $\nu \in \mathbb{R}_{+}$

$$
\left.I\right|_{t=0}=I_{0}(x, \omega, \nu) \mid \begin{array}{r}
I_{\infty}(\omega, \nu) \quad \text { for } x<-N \\
I^{0}(x, \omega, \nu) \text { for }|x| \leqslant N \\
I_{\infty}(\omega, \nu) \text { for } x>N
\end{array}
$$

with $N>0, U^{0}$ and $I^{0}$ are measurable functions, $U_{\infty}$ is a constant state, $I_{\infty}=B\left(\nu, \vartheta_{\infty}\right)$ where $\vartheta_{\infty}$ is the temperature associated with $U_{\infty}$.

Denoting by $\eta:=\rho^{-1}$ the specific volume, pressure $p(\rho, S)$, internal energy $e(\rho, S)$ and temperature $\vartheta(\rho, S)$ are related by the thermodynamical relations

$$
p=-\partial_{\eta} e \text { and } \vartheta=\partial_{S} e .
$$

We assume that state functions $e$ and $p$ (resp. $\sigma_{a}$ and $\sigma_{s}$ ) are $C^{2}$ (resp $C^{1}$ ) functions of their arguments and we suppose that $e$ satisfies the following stability conditions

$$
\left\{\begin{array}{c}
e(\eta, S)>0, \quad \partial_{\eta} e(\eta, S)<0, \quad \partial_{S} e(\eta, S)>0 \\
\partial_{\eta \eta}^{2} e(\eta, S)>0, \quad \partial_{\eta S}^{2} e(\eta, S)<0, \quad \partial_{\eta \eta \eta}^{3} e(\eta, S)<0 \\
\lim _{S \rightarrow+\infty} e(\eta, S)=+\infty, \quad \lim _{S \rightarrow-\infty} \partial_{\eta} e(\eta, S)=0
\end{array}\right.
$$

These conditions imply that for any couple $(\eta, p)$, there is a unique $S:=S(\eta, p)$ such that $\partial_{\eta} e(\eta, S)=-p$ and we note $\varepsilon(\eta, p):=e(\eta, S(\eta, p))$. After Smith [30], we assume that

$$
\lim _{\eta \rightarrow 0} \varepsilon(\eta, p)=0,\left.\quad \partial_{\eta} p\right|_{\varepsilon} \leqslant \frac{p^{2}}{2 \varepsilon} .
$$

Here, $\left.\partial_{\eta} p\right|_{\varepsilon}$ denotes the partial derivative of $p=p(\eta, \varepsilon)$ as a function of $\eta=1 / \rho$ and $\varepsilon=e$.

Finally, we give the conditions we need on $\sigma_{a}$ and $\sigma_{s}$ : we assume that

$$
\begin{gathered}
\exists h_{a} \in C^{0}\left[(0, \infty)^{2}\right], \quad \sup _{\nu \in \mathbb{R}_{+}} \sigma_{a}(\nu, \rho, \vartheta) \leqslant h_{a}(\rho, \vartheta) \overline{\sigma_{a}}(t), \\
\exists h_{s} \in C^{0}\left[(0, \infty)^{2}\right], \quad \sup _{\nu \in \mathbb{R}_{+}} \sigma_{s}(\nu, \rho, \vartheta) \leqslant h_{s}(\rho, \vartheta) \overline{\sigma_{s}}(t), \\
\exists \tilde{h}_{a} \in C^{0}\left[(0, \infty)^{2}\right], \quad \sup _{\nu \in \mathbb{R}_{+}}\left(\left|\partial_{\rho} \sigma_{a}(\nu, \rho, \vartheta)\right|+\left|\partial_{\vartheta} \sigma_{a}(\nu, \rho, \vartheta)\right|\right) \leqslant \tilde{h}_{a}(\rho, \vartheta) \overline{\sigma_{a}}(t),
\end{gathered}
$$

and

$$
\exists \tilde{h}_{s} \in C^{0}\left[(0, \infty)^{2}\right], \quad \sup _{\nu \in \mathbb{R}_{+}}\left(\left|\partial_{\rho} \sigma_{s}(\nu, \rho, \vartheta)\right|+\left|\partial_{\vartheta} \sigma_{s}(\nu, \rho, \vartheta)\right|\right) \leqslant \tilde{h}_{s}(\rho, \vartheta) \overline{\sigma_{s}}(t) .
$$

Here, $C^{0}$ means continuous, and $\overline{\sigma_{a}}$ and $\overline{\sigma_{s}}$ are supposed to be in $L^{1}\left(\mathbb{R}^{+}\right) \cap C^{0}\left(\mathbb{R}^{+}\right)$. A simple argument then proves that

Lemma 1. Assume that (21) is satisfied, and that $g$ is defined by (5). Then, there exists $h_{0} \in C^{0}[(0, \infty) \times[0, \infty)]$, such that, for any $I \geqslant 0$ in $L_{\omega, \nu}^{1}\left([-1,1] \times \mathbb{R}^{+}\right)$and any $U \in \mathbb{R}_{+} \times \mathbb{R} \times \mathbb{R}_{+}$,

$$
\int_{0}^{\infty} \int_{-1}^{1}|\mathcal{S}(I, U)| d \omega d \nu \leqslant h_{0}\left(|U|,\|I\|_{L_{\omega, \nu}^{1}\left([-1,1] \times \mathbb{R}^{+}\right)}\right)\left(\overline{\sigma_{a}}(t)+\overline{\sigma_{s}}(t)\right) .
$$

Moreover, there exists $h_{1} \in C^{0}[(0, \infty) \times[0, \infty)]$, such that, for any $I \geqslant 0$ in $L^{1}\left(\mathbb{R}_{+} \times[-1,1]\right)$ and any $U \in$ $\mathbb{R}_{+} \times \mathbb{R} \times \mathbb{R}_{+}$,

$$
|g(I, U)| \leqslant h_{1}\left(|U|,\|I\|_{L_{\omega, \nu}^{1}\left([-1,1] \times \mathbb{R}^{+}\right)}\right)\left(\overline{\sigma_{a}}(t)+\overline{\sigma_{s}}(t)\right) .
$$

Finally, if in addition (23) is satisfied, there exists $h_{2} \in C^{0}\left[(0, \infty)^{2} \times[0, \infty)^{2}\right]$ such that for any $I_{1}, I_{2} \geqslant 0$ in $L^{1}\left(\mathbb{R}_{+} \times[-1,1]\right)$ and any $\left(U_{1}, U_{2}\right) \in\left(\mathbb{R}_{+} \times \mathbb{R} \times \mathbb{R}_{+}\right)^{2}$,

$$
\begin{aligned}
\left|g\left(I_{1}, U_{1}\right)-g\left(I_{2}, U_{2}\right)\right| \leqslant h_{2}\left(\left|U_{1}\right|,\left|U_{2}\right|,\left\|I_{1}\right\|_{L^{1}},\right. & \left.\left\|I_{2}\right\|_{L_{\omega, \nu}^{1}\left([-1,1] \times \mathbb{R}^{+}\right)}\right) \\
& \times\left[\left|U_{1}-U_{2}\right|+\left\|I_{1}-I_{2}\right\|_{L_{\omega, \nu}^{1}\left([-1,1] \times \mathbb{R}^{+}\right)}\right]\left(\overline{\sigma_{a}}(t)+\overline{\sigma_{s}}(t)\right) .
\end{aligned}
$$


Proof: Recall that, according to $(7), \mathcal{S}=\sigma_{a}(B-I)+\sigma_{s}(\tilde{I}-I)$, hence, applying (21) and (22),

$$
\int_{0}^{\infty} \int_{-1}^{1}|\mathcal{S}|(x, t, \nu, \omega) d \omega d \nu \leqslant \int_{0}^{\infty} \int_{-1}^{1} \overline{\sigma_{a}}(t) h_{a}(\rho, \vartheta)|B-I| d \omega d \nu+\int_{0}^{\infty} \int_{-1}^{1} \overline{\sigma_{s}}(t) h_{s}(\rho, \vartheta)|\tilde{I}-I| d \omega d \nu .
$$

Next, we use the fact that $\int_{0}^{\infty} B d \nu=a \vartheta^{4}$, for some pure constant $a>0$, finding

$$
\int_{0}^{\infty} \int_{-1}^{1}|\mathcal{S}|(x, t, \nu, \omega) d \omega d \nu \leqslant \overline{\sigma_{a}}(t) h_{a}(\rho, \vartheta)\left(a \vartheta^{4}+\|I\|_{L_{\omega, \nu}^{1}\left([-1,1] \times \mathbb{R}^{+}\right)}\right)+2 \overline{\sigma_{s}}(t) h_{s}(\rho, \vartheta)\|I\|_{L_{\omega, \nu}^{1}\left([-1,1] \times \mathbb{R}^{+}\right)} .
$$

This gives (25). Next, we have

$$
|g| \leqslant \frac{1}{\rho} \int_{0}^{\infty} \int_{-1}^{1}|\mathcal{S}|+2 \frac{|v|}{\rho \vartheta} \int_{0}^{\infty} \int_{-1}^{1}|\mathcal{S}|,
$$

hence (26). As for (27), the same kind of proof applies.

\section{The approximating scheme}

The idea is first to freeze the unknown $U$ in the second equation of (15) which allows to find $I$ as the solution of a linear Boltzmann's equation. Then plugging $I$ into the quasilinear hyperbolic part (first equation (15)) we get $U$ by solving this system by using a discrete scheme mixing Glimm-Liu scheme for the conservative part and a fractional step method for the source term, using ideas of T.-P. Liu [23], Hong-Lefloch [12] and Dafermos-Hsiao $[6]$.

\subsection{An iterative method}

In order to achieve this program, we first consider the family $\left(U^{\ell}, I^{\ell}\right) \equiv\left(\rho^{\ell}, v^{\ell}, S^{\ell}, I^{\ell}\right)$ defined inductively for $\ell>1$ by

$$
\left\{\begin{array}{c}
\partial_{t} U^{\ell}(x, t)+\partial_{x} f\left(U^{\ell}(x, t)\right)=g^{\ell}, \\
\frac{1}{c} \partial_{t} I^{\ell}(x, t, \nu, \omega)+\omega \partial_{x} I^{\ell}(x, t, \nu, \omega)=\mathcal{S}^{\ell},
\end{array}\right.
$$

for $(x, t) \in \mathbb{R} \times \mathbb{R}_{+}$, where

$$
g^{\ell}=\left(\begin{array}{c}
0 \\
-\frac{1}{\rho^{\ell}} \mathcal{S}_{F}^{\ell} \\
\frac{1}{\rho^{\ell} \vartheta^{\ell}}\left(v^{\ell} \mathcal{S}_{F}^{\ell}-\mathcal{S}_{E}^{\ell}\right)
\end{array}\right),
$$

with

$$
\mathcal{S}_{E}^{\ell}:=\int_{-1}^{1} \int_{0}^{\infty} \mathcal{S}\left(U^{\ell-1}, I^{\ell}\right) d \nu d \omega, \quad \mathcal{S}_{F}^{\ell}:=\frac{1}{c} \int_{-1}^{1} \int_{0}^{\infty} \omega \mathcal{S}\left(U^{\ell-1}, I^{\ell}\right) d \nu d \omega,
$$

for

$$
\mathcal{S}(V, W)=\sigma_{a}(t, \nu, V)[B(\nu, V)-W]+\sigma_{s}(t, \nu, V)[\tilde{W}-W]
$$

and

$$
\mathcal{S}^{\ell}=\mathcal{S}\left(U^{\ell-1}, I^{\ell}\right)
$$

with initial conditions

$$
\left.U^{\ell}\right|_{t=0}=U_{0}(x)
$$

and

$$
\left.I^{\ell}\right|_{t=0}=I_{0}(x, \nu, \omega)
$$

We define the sequence $\left\{\left(U^{\ell}, I^{\ell}\right)\right\}_{\ell \geqslant 1}$ as follows. 
1. Solving first the linear Boltzmann's equation

$$
\frac{1}{c} \partial_{t} I^{1}(x, t, \nu, \omega)+\omega \partial_{x} I^{1}(x, t, \nu, \omega)=\mathcal{S}^{1},
$$

with

$$
\left.I^{1}\right|_{t=0}=I_{0}(x, \nu, \omega)
$$

and

$$
\mathcal{S}^{1}=\mathcal{S}\left(U_{0}, I^{1}\right),
$$

gives $I^{1}(x, t, \nu, \omega)$ for $x \in \mathbb{R}$ and $0<t \leqslant \Delta t$.

2. Then solving the hyperbolic system

$$
\partial_{t} U^{1}(x, t)+\partial_{x}\left(f\left(U^{1}(x, t)\right)\right)=g^{1},
$$

with

$$
\left.U^{1}\right|_{t=0}=U_{0}(x)
$$

defines $U^{1}(x, t)$ for $x \in \mathbb{R}$ and $0<t \leqslant \Delta t$.

3. Supposing now that for any $\ell>1$, we know $\left(U^{\ell-1}, I^{\ell-1}\right)$ for $x \in \mathbb{R}$ and $(\ell-2) \Delta t<t \leqslant(\ell-1) \Delta t$, we solve the linear Boltzmann's equation

$$
\frac{1}{c} \partial_{t} I^{\ell}(x, t, \nu, \omega)+\omega \partial_{x} I^{\ell}(x, t, \nu, \omega)=\mathcal{S}\left(\tilde{U}^{\ell}, I^{\ell}\right)
$$

with source

$$
\tilde{U}^{\ell}(x, t)=U^{\ell-1}(x,(\ell-1) \Delta t),
$$

and initial data

$$
\left.I^{\ell}\right|_{t=(\ell-1) \Delta t}=I^{\ell-1}(x,(\ell-1) \Delta t, \nu, \omega),
$$

which gives $I^{\ell}(x, t, \nu, \omega)$ for $x \in \mathbb{R}$ and $(\ell-1) \Delta t<t \leqslant \ell \Delta t$.

4. Plugging then $I^{\ell}(x, t, \nu, \omega)$ in the right-hand side, we solve the hyperbolic system

$$
\partial_{t} U^{\ell}(x, t)+\partial_{x}\left(f\left(U^{\ell}(x, t)\right)\right)=g^{\ell},
$$

with

$$
\left.U^{\ell}\right|_{t=\ell \Delta t}=U^{\ell-1}(x, \ell \Delta t)
$$

which defines $U^{\ell}(x, t)$ for $x \in \mathbb{R}$ and $(\ell-1) \Delta t<t \leqslant \ell \Delta t$.

\subsection{The Riemann problem for the radiative transfer equation}

We consider for $(x, t, \omega, \nu) \in \mathbb{R} \times[0, T] \times[-1,1] \times \mathbb{R}_{+}$the problem

$$
\left\{\begin{array}{c}
\partial_{t} I(x, t, \nu, \omega)+c \omega \partial_{x} I(x, t, \nu, \omega)=c \mathcal{S} \text { for } t>t_{0}, \\
\left.I\right|_{t=0} \equiv I^{i n}(x, \omega, \nu)=\mid \begin{array}{c}
I_{L}(\omega, \nu) \text { for } x<x_{0}, \\
I_{R}(\omega, \nu) \text { for } x>x_{0},
\end{array},
\end{array}\right.
$$

where $t_{0}>0$,

$$
\begin{gathered}
\mathcal{S}=\sigma_{a}(\nu, U)[B(\nu, U)-I(x, t, \nu, \omega)]+\sigma_{s}(\nu, U)[\tilde{I}(x, t, \nu)-I(x, t, \nu, \omega)], \\
\tilde{I}(x, t, \nu):=\frac{1}{2} \int_{-1}^{1} I(x, t, \nu, \omega) d \omega,
\end{gathered}
$$

and

and

$$
B(\nu, U)=2 h \nu^{3} c^{-2}\left(e^{\frac{h \nu}{k^{\vartheta} \vartheta}}-1\right)^{-1}
$$

$$
U(x, t)=\mid \begin{aligned}
& U_{L} \text { for } x<x_{0} \\
& U_{R} \text { for } x>x_{0}
\end{aligned}
$$

The following standard result holds (see [8] and [10]). 
Proposition 1. Suppose that $I^{\text {in }} \in L^{\infty}\left(\mathbb{R} \times[-1,1] \times \mathbb{R}_{+}\right)$and that

$(x, t, \omega, \nu) \rightarrow a(x, t, \omega, \nu):=\sigma_{a}(\nu, \omega, \rho(x, t), \vartheta(x, t))+\sigma_{s}(\nu, \omega, \rho(x, t), \vartheta(x, t)) \in L^{\infty}\left(\mathbb{R} \times[0, T] \times[-1,1] \times \mathbb{R}_{+}\right)$,

where $\sigma_{a} \geqslant 0$ and $\sigma_{s} \geqslant 0$.

The problem (31) has a unique generalized solution $I \in L^{\infty}\left(\mathbb{R} \times[0, T] \times[-1,1] \times \mathbb{R}_{+}\right)$.

Morever suppose that

$$
(x, t, \omega, \nu) \rightarrow Q(x, t, \omega, \nu):=\sigma_{a}(\nu, \omega, \rho(x, t), \vartheta(x, t)) B(\nu, \vartheta(x, t)) \in L^{\infty}\left(\mathbb{R} \times[0, T] \times[-1,1] \times \mathbb{R}_{+}\right) .
$$

The following bound holds

$$
I(x, t, \omega, \nu) \leqslant\left\|I^{i n}\right\|_{L^{\infty}\left(\mathbb{R} \times[-1,1] \times \mathbb{R}_{+}\right)}+T\|Q\|_{L^{\infty}\left(\mathbb{R} \times[0, T] \times[-1,1] \times \mathbb{R}_{+}\right)} .
$$

Proof: Although this classical result is well-known, it is not easy to find it in the literature. We therefore provide a proof, borrowed from [10], which uses only elementary arguments. Applying the method of characteristics to the transport equation (31) and using the notations

$$
\begin{gathered}
\mathcal{A}(x, t, \nu):=c\left(\sigma_{a}(\nu, U(t, x))+\sigma_{s}(\nu, U(t, x))\right), \\
\mathcal{K} I(x, t, \omega, \nu):=\frac{c}{2} \sigma_{s}(\nu, U(t, x)) \int_{-1}^{1} I(x, t, \omega, \nu) d \omega,
\end{gathered}
$$

and

$$
\mathcal{Q}(x, t, \nu):=c \sigma_{a}(\nu, U(t, x)) B(\nu, U(t, x)),
$$

for any $(x, t, \omega, \nu) \in\left(\mathbb{R} \times[0, T] \times[-1,1] \times \mathbb{R}_{+}\right)$and for $I \in L^{\infty}\left(\mathbb{R} \times[0, T] \times[-1,1] \times \mathbb{R}_{+}\right)$, one checks the formula

$$
I=\mathcal{F}\left[I^{i n}, Q\right]+\mathcal{T} I
$$

where

$$
\begin{aligned}
\mathcal{F}\left[I^{i n}, Q\right](x, t, \omega, \nu)=I^{i n}(x-c \omega t, \omega, \nu) e^{-\int_{0}^{t} \mathcal{A}(x+c(\tau-t) \omega, \tau, \omega, \nu) d \tau} & \\
& +\int_{0}^{t} \mathcal{Q}(x+c(s-t) \omega, s, \omega, \nu) e^{-\int_{s}^{t} \mathcal{A}(x+c(\tau-t) \omega, \tau, \omega, \nu) d \tau} d s
\end{aligned}
$$

and

$$
\mathcal{T} I(x, t, \omega, \nu)=\int_{0}^{t} \mathcal{K} I(x+c(s-t) \omega, s, \omega, \nu) e^{-\int_{s}^{t} \mathcal{A}(x+c(\tau-t) \omega, \tau, \omega, \nu) d \tau} d s .
$$

One also checks in the same stroke

$$
I(x, t, \omega, \nu)=I^{i n}(x-c \omega t, \omega, \nu)+\int_{0}^{t}(\mathcal{K} I+\mathcal{Q}-\mathcal{A} I)(x+c(s-t) \omega, s, \omega, \nu) d s
$$

Considering (33) as a fixed point equation, one is led to show the convergence of the series in

$$
I:=\sum_{n \geqslant 0} \mathcal{T}^{n} \mathcal{F}\left[I^{i n}, Q\right]
$$

We denote by $\mathcal{U}=\left\{U(x, t), x \in \mathbb{R}, t \in \mathbb{R}_{+}\right\}$. One has first

$$
\left\|\mathcal{F}\left[I^{i n}, Q\right]\right\|_{L^{\infty}(\mathbb{R} \times[-1,1] \times \mathcal{U})} \leqslant\left\|I^{i n}(\cdot, \omega, \nu)\right\|_{L^{\infty}(\mathbb{R} \times[-1,1] \times \mathcal{U})}+T\|\mathcal{Q}\|_{L^{\infty}(\mathbb{R} \times[-1,1] \times \mathcal{U})} .
$$

Moreover, we have, for any $J \in L^{\infty}\left(\mathbb{R} \times[0, T] \times[-1,1] \times \mathbb{R}^{+}\right)$,

$$
|\mathcal{K} J(x, t, \omega, \nu)| \leqslant \underbrace{\left(c \sup _{\nu>0, U \in \mathcal{U}} \sigma_{s}(\nu, U)\right)}_{M(\mathcal{U})} \sup _{\omega^{\prime} \in[-1,1]}\left|J\left(x, t, \omega^{\prime}, \nu\right)\right| .
$$


Hence, we have

$$
\begin{aligned}
\left|\mathcal{T}^{n} J\right|(x, t, \omega, \nu) & \leqslant \int_{0}^{t} M(\mathcal{U}) \sup _{\omega^{\prime} \in[-1,1]}\left|\mathcal{T}^{n-1} J\left(x+c(s-t) \omega^{\prime}, s, \omega^{\prime}, \nu\right)\right| d s \\
& \leqslant M(\mathcal{U}) \int_{0}^{t} \sup _{\left(x^{\prime}, \omega^{\prime}, \nu^{\prime}\right) \in \mathbb{R} \times[-1,1] \times \mathbb{R}^{+}}\left|\mathcal{T}^{n-1} J\left(x^{\prime}, s, \omega^{\prime}, \nu^{\prime}\right)\right| d s \\
& \leqslant M(\mathcal{U})^{n} \int_{0}^{t} \int_{0}^{t_{1}} \ldots \int_{0}^{t_{n-1}} \sup _{\left(x^{\prime}, \omega^{\prime}, \nu^{\prime}\right) \in \mathbb{R} \times[-1,1] \times \mathbb{R}^{+}}\left|J\left(x^{\prime}, t_{n}, \omega^{\prime}, \nu^{\prime}\right)\right| d t_{n} \ldots d t_{1} \\
& \leqslant \frac{M(\mathcal{U})^{n} t^{n}}{n !}\|J\|_{L^{\infty}\left(\mathbb{R} \times[0, T] \times[-1,1] \times \mathbb{R}_{+}\right)} .
\end{aligned}
$$

Applying this inequality to $J=\mathcal{F}\left[I^{i n}, Q\right]$, and summing over $n$, we thus have:

$$
\sum_{n \geqslant 0}\left\|\mathcal{T}^{n} \mathcal{F}\left[I^{i n}, Q\right]\right\|_{L^{\infty}\left(\mathbb{R} \times[0, T] \times[-1,1] \times \mathbb{R}_{+}\right)} \leqslant \sum_{n \geqslant 0} \frac{M(\mathcal{U})^{n} T^{n}}{n !}\left\|\mathcal{F}\left[I^{i n}, Q\right]\right\|_{L^{\infty}\left(\mathbb{R} \times[0, T] \times[-1,1] \times \mathbb{R}_{+}\right)} .
$$

Hence, the sum (35) is normally convergent in $L^{\infty}\left(\mathbb{R} \times[0, T] \times[-1,1] \times \mathbb{R}_{+}\right)$. Moreover, if $I^{\text {in }} \geqslant 0$ and $Q \geqslant 0$, then $\mathcal{F}\left[I^{i n}, Q\right] \geqslant 0$. Since, in addition, $J \geqslant 0$ implies $\mathcal{T}(J) \geqslant 0$, we infer that each term of the series $(35)$ is non-negative, so

$$
\left(I^{i n} \geqslant 0, Q \geqslant 0\right) \quad \Rightarrow \quad I \geqslant 0
$$

Now, defining $Z=\left\|I^{i n}\right\|_{L^{\infty}\left(\mathbb{R} \times[-1,1] \times \mathbb{R}_{+}\right)}+t\|Q\|_{L^{\infty}\left(\mathbb{R} \times[0, T] \times \mathbb{R}_{+}\right)}-I$, we clearly have

$$
\begin{aligned}
\partial_{t} Z+c \omega \partial_{x} Z-\mathcal{K} Z+\mathcal{A} Z= & -Q+\|Q\|_{L^{\infty}\left(\mathbb{R} \times[0, T] \times \mathbb{R}_{+}\right)} \\
& -\mathcal{K}\left(\left\|I^{i n}\right\|_{L^{\infty}\left(\mathbb{R} \times[-1,1] \times \mathbb{R}_{+}\right)}+t\|Q\|_{L^{\infty}\left(\mathbb{R} \times[0, T] \times \mathbb{R}_{+}\right)}\right) \\
& +\mathcal{A}\left(\left\|I^{i n}\right\|_{L^{\infty}\left(\mathbb{R} \times[-1,1] \times \mathbb{R}_{+}\right)}+t\|Q\|_{L^{\infty}\left(\mathbb{R} \times[0, T] \times \mathbb{R}_{+}\right)}\right) \\
\geqslant & 0,
\end{aligned}
$$

where we have used the fact that $\left\|I^{i n}\right\|_{L^{\infty}\left(\mathbb{R} \times[-1,1] \times \mathbb{R}_{+}\right)}+t\|Q\|_{L^{\infty}\left(\mathbb{R} \times[0, T] \times \mathbb{R}_{+}\right)}$is independent of $\omega$. Hence, applying (36) to $Z$, we infer $Z \geqslant 0$, that is, (32).

\subsection{The generalized Riemann problem for radiative hydrodynamics}

Given two states $\left(U_{L}, I_{L}\right)\left(U_{R}, I_{R}\right)$ and a point $\left(x_{0}, t_{0}\right)$, we consider now the generalized Riemann problem $G R\left(x_{0}, t_{0} ; U_{L}, I_{L}, U_{R}, I_{R}\right)$

$$
\left\{\begin{array}{c}
\partial_{t} U+\partial_{x} f(U)=g(I, U) \quad \text { for } t>t_{0}, \quad x \in \mathbb{R}, \\
\partial_{t} I+c \omega \partial_{x} I=c \mathcal{S}(I, U) \quad \text { for } t>t_{0}, \quad x \in \mathbb{R}, \quad(\omega, \nu) \in \times[-1,1] \times \mathbb{R}_{+} \\
\left(U\left(x, t_{0}\right), I\left(x, t_{0}\right)\right)=\mid \begin{array}{l}
\left(U_{L}, I_{L}\right) \text { for } x<x_{0}, \\
\left(U_{R}, I_{R}\right) \text { for } x>x_{0} .
\end{array}
\end{array}\right.
$$

After [12] we treat the hydrodynamical part of (37) as a perturbation of the classical Riemann problem $C R U\left(x_{0}, t_{0} ; U_{L}, U_{R}\right)$

$$
\left\{\begin{array}{c}
\partial_{t} U+\partial_{x} f(U)=0 \text { for } t>t_{0}, \quad x \in \mathbb{R} \\
U(x, 0)=\mid \begin{array}{c}
U_{L} \text { for } x<x_{0}, \\
U_{R} \text { for } x>x_{0},
\end{array}
\end{array}\right.
$$

for $(x, t) \in \mathbb{R} \times \mathbb{R}_{+}$.

From general results (see Serre [29] and references therein) one knows that $C R U\left(x_{0}, t_{0} ; U_{L}, U_{R}\right)$ has a selfsimilar solution $W_{C}\left(\xi, x_{0}, t_{0} ; U_{L}, U_{R}\right)$ with $\xi=\frac{x-x_{0}}{t-t_{0}}$ provided that the quantity $\left|U_{R}-U_{L}\right|$ is small enough, which consists of at most 4 constant states $U_{i} i \leqslant 4$ separated by shock waves, contact discontinuities or rarefaction waves. We say that $C R\left(x_{0}, t_{0} ; U_{L}, U_{R}\right)$ is solved by the elementary waves $\left(U_{i-1}, U_{i}\right)$ with $i=1, \ldots, 4$ if each $U_{i}$ 
belongs to the $i$-wave curve $\mathcal{W}_{i}\left(U_{i-1}\right)$ issued from the state $U_{i-1}$ in phase space and $\left(U_{i-1}, U_{i}\right)$ is called an $i$-wave of $C R\left(x_{0}, t_{0} ; U_{L}, U_{R}\right)$.

If the $i$-characteristic field is genuinely nonlinear then $\mathcal{W}_{i}\left(U_{i-1}\right)$ consists in two parts: the $i$-rarefaction curve and the $i$-shock issued from $U_{i-1}$. If the $i$-characteristic field is linearly degenerate then $\mathcal{W}_{i}\left(U_{i-1}\right)$ consists in a $C^{2}$ curve of $i$-contact discontinuities.

Denoting by $\varepsilon_{i} \equiv \varepsilon_{i}\left(U_{L}, U_{R} ; t_{0}, x_{0}\right)$ the strength of the $i$-wave $\left(U_{i-1}, U_{i}\right)$ along the $i$-curve, one can assume that, if the $i$-characteristic field is genuinely nonlinear then $\varepsilon_{i} \geqslant 0$ for an $i$-rarefaction curve and $\varepsilon_{i} \leqslant 0$ for an $i$-shock. The global strength of $W_{C}\left(\xi, x_{0}, t_{0} ; U_{L}, U_{R}\right)$ is then the vector $\varepsilon=\left(\varepsilon_{1}, \varepsilon_{2}, \varepsilon_{3}\right)$.

We also denote by $\sigma_{i}^{-}=\lambda_{i}\left(U_{i-1}, t_{0}, x_{0}\right)$ and $\sigma_{i}^{+}=\lambda_{i}\left(U_{i}, t_{0}, x_{0}\right)$ the lower and upper speeds of the $i-$ wave $\left(U_{i-1}, U_{i}\right)$ when it is a rarefaction and just by $\sigma_{i}$ the speed of $\left(U_{i-1}, U_{i}\right)$ if it is an $i$-shock or an $i-$ contact discontinuity.

We also treat the radiative part of $(37)$ as a perturbation of the linear problem $C R I\left(x_{0}, t_{0} ; I_{L}, I_{R}\right)$

$$
\left\{\begin{array}{c}
\partial_{t} I(x, t, \nu, \omega)+c \omega \partial_{x} I(x, t, \nu, \omega)=0 \text { for } t>t_{0}, \\
\left.I\right|_{t=0} \equiv I^{i n}(x, \omega, \nu)=\mid \begin{array}{c}
I_{L}(\omega, \nu) \text { for } x<x_{0} \\
I_{R}(\omega, \nu) \text { for } x>x_{0}
\end{array}
\end{array}\right.
$$

the explicit solution of which is

$$
I_{C}(x, t, \omega, \nu)=I^{i n}(x-c \omega t, \omega, \nu),
$$

and one observes that it rewrites as $I_{C}\left(\xi, \omega, \nu, x_{0}, t_{0} ; I_{L}, I_{R}\right)$.

In this trivial case there is only one (linearly degenerate) field and one can decide that the strength of the unique simple wave is

$$
\varepsilon_{r} \equiv \varepsilon_{r}\left(I_{L}, I_{R}, \omega, \nu ; t_{0}, x_{0}\right)=I_{R}-I_{L}
$$

A straightforward extension of [19] shows now that the generalized Riemann problem $G R\left(x_{0}, t_{0} ; U_{L}, I_{L}, U_{R}, I_{R}\right)$ has also a solution piecewise smooth, locally similar to $\left(W_{C}\left(\xi, x_{0}, t_{0} ; U_{L}, U_{R}\right), I_{C}\left(\xi, \omega, \nu, x_{0}, t_{0} ; I_{L}, I_{R}\right)\right)$.

After [12], we define our approximate solution $\left(W_{G}, I_{G}\right)$ of the generalized Riemann problem $G R\left(x_{0}, t_{0} ; U_{L}, I_{L}, U_{R}, I_{R}\right)$ by the perturbative expansion

$$
W_{G}\left(t, x ; x_{0}, t_{0} ; U_{L}, U_{R}\right)=W_{C}\left(\xi, x_{0}, t_{0} ; U_{L}, U_{R}\right)+\left(t-t_{0}\right) g\left(I_{C}\left(t, \omega, \nu, t_{0}, x_{0} ; I_{L}, I_{R}\right), W_{C}\left(t, x_{0}, t_{0} ; U_{L}, U_{R}\right)\right)
$$

for $t>t_{0}$ and $x \in \mathbb{R}$, where the correction is small when $t-t_{0}$ is small, and in the same way

$$
\begin{gathered}
I_{G}\left(t, x, \omega, \nu ; x_{0}, t_{0} ; I_{L}, I_{R}\right)=I_{C}\left(\xi, \omega, \nu, x_{0}, t_{0} ; I_{L}, I_{R}\right) \\
+c\left(t-t_{0}\right) \mathcal{S}\left(I_{C}\left(t, \omega, \nu, t_{0}, x_{0} ; I_{L}, I_{R}\right), W_{C}\left(t, x_{0}, t_{0} ; U_{L}, U_{R}\right)\right),
\end{gathered}
$$

We will use the notation $W_{G}(t, x)\left(\operatorname{resp} I_{G}(t, x, \omega, \nu)\right)$ for $W_{G}\left(t, x ; x_{0}, t_{0} ; U_{L}, U_{R}\right)\left(\operatorname{resp} . I_{G}\left(t, x, \omega, \nu ; x_{0}, t_{0} ; I_{L}, I_{R}\right)\right.$.

Definition 1. In the sequel, we assume that $\left(\rho_{\infty}, v_{\infty}, S_{\infty}, I_{\infty}\right)$ is a steady state for the system $(2)$, and that $\mathcal{U}$ is a small neighborhood of $\left(\rho_{\infty}, v_{\infty}, S_{\infty}, I_{\infty}\right)$ in $\mathbb{R}^{4}$.

Given space and time steps $\Delta t$ and $\Delta x$ satisfying the CFL condition

$$
\frac{\Delta t}{\Delta x} \max \left[\max _{i=1,2,3}\left(\sup _{u \in \mathcal{U}}\left|\lambda_{i}(u)\right|\right), c\right] \leqslant 1,
$$

we show first that actually $\left(W_{G}, I_{G}\right)$ solves approximately $G R$.

Proposition 2. Suppose $\phi \in X:=C^{1}\left(\mathbb{R}_{+} \times \mathbb{R}, \mathbb{R}^{3}\right)$ and $\psi \in Y:=C^{1}\left(\mathbb{R}_{+} \times \mathbb{R}, \mathbb{R}\right)$ are two compactly supported functions in the strip $(0, \Delta t)$. Then for any $\left(t_{0}, x_{0}\right) \in \mathbb{R}_{+} \times \mathbb{R},\left(U_{L}, I_{L}\right),\left(U_{R}, I_{R}\right) \in \mathcal{U}^{2}$ and for any $\Delta t$ and $\Delta x$ satisfying the condition (42) 
1. the function $W_{G}\left(t, x ; x_{0}, t_{0} ; U_{L}, U_{R}\right)$ satisfies

$$
\begin{gathered}
\int_{t_{0}}^{t_{0}+\Delta t} \int_{x_{0}-\Delta x}^{x_{0}+\Delta x}\left\{W_{G} \partial_{t} \phi+f\left(W_{G}\right) \partial_{x} \phi+g\left(I_{G}, W_{G}\right) \phi\right\} d x d t \\
=\int_{x_{0}-\Delta x}^{x_{0}+\Delta x} W_{G}\left(t_{0}+\Delta t, \cdot\right) \phi\left(t_{0}+\Delta t, \cdot\right) d x-\int_{x_{0}-\Delta x}^{x_{0}+\Delta x} W_{G}\left(t_{0}, \cdot\right) \phi\left(t_{0}, \cdot\right) d x \\
+\int_{t_{0}}^{t_{0}+\Delta t} f\left(W_{G}\left(\cdot, x_{0}+\Delta x\right)\right) \phi\left(\cdot, x_{0}+\Delta x\right) d t-\int_{t_{0}}^{t_{0}+\Delta t} f\left(W_{G}\left(\cdot, x_{0}-\Delta x\right)\right) \phi\left(\cdot, x_{0}-\Delta x\right) d t \\
+O(1)\left(\Delta t^{2}\left(\Delta t+\Delta x+\left|U_{R}-U_{L}\right|\right)\right)\|\phi\|_{X} .
\end{gathered}
$$

2. the function $I_{G}\left(t, x, \omega, \nu ; x_{0}, t_{0}\right)$ satisfies

$$
\begin{gathered}
\int_{t_{0}}^{t_{0}+\Delta t} \int_{x_{0}-\Delta x}^{x_{0}+\Delta x}\left\{I_{G} \partial_{t} \psi+c \omega I_{G} \partial_{x} \psi+c \mathcal{S}\left(I_{G}, W_{G}\right) \psi\right\} d x d t \\
=\int_{x_{0}-\Delta x}^{x_{0}+\Delta x} I_{G}\left(t_{0}+\Delta t, \cdot\right) \psi\left(t_{0}+\Delta t, \cdot\right) d x-\int_{x_{0}-\Delta x}^{x_{0}+\Delta x} I_{G}\left(t_{0}, \cdot\right) \psi\left(t_{0}, \cdot\right) d x \\
+\int_{t_{0}}^{t_{0}+\Delta t} c \omega I_{G}\left(\cdot, x_{0}+\Delta x\right) \psi\left(\cdot, x_{0}+\Delta x\right) d t-\int_{t_{0}}^{t_{0}+\Delta t} c \omega I_{G}\left(\cdot, x_{0}-\Delta x\right) \psi\left(\cdot, x_{0}-\Delta x\right) d t \\
+O(1)(\Delta t)^{2}(\Delta t+\Delta x)\|\psi\|_{Y} .
\end{gathered}
$$

Proof: As we essentially follow the proof of Hong and LeFloch (see Proposition 2.1 in [12]) we will sketch the arguments only emphasizing the structure of radiation sources through $I$.

Assuming that $\left(t_{0}, x_{0}\right)=(0,0)$ and defining the auxiliary function $M(t, x):=W_{G} \partial_{t} \phi+f\left(W_{G}\right) \partial_{x} \phi+$ $g\left(I_{G}, W_{G}\right) \phi$, we call $I_{1}$ the subset of indices $i$ such that $\forall i \in I_{1}$ the corresponding $i$-wave is either a shock or a contact discontinuity, and $I_{2}$ the subset of indices $i$ such that $\forall i \in I_{2}$ the corresponding $i$-wave is a rarefaction wave (clearly $I_{1} \cup I_{2}=\{1,2,3\}$ ). We have the following decomposition of $\iint M d x d t$ on the elementary cell $[0, \Delta t] \times[-\Delta x, \Delta x]$

$$
\begin{gathered}
\int_{0}^{\Delta t} \int_{-\Delta x}^{\Delta x} M(t, x) d x d t=\sum_{i \in I_{1}} \int_{0}^{\Delta t} \int_{\sigma_{i}^{+} t}^{\sigma_{i+1}^{-} t} M(t, x) d x d t \\
+\int_{0}^{\Delta t} \int_{-\Delta x}^{\sigma_{1}^{-} t} M(t, x) d x d t+\int_{0}^{\Delta t} \int_{\sigma_{3}^{+} t}^{\Delta x} M(t, x) d x d t \\
\quad+\sum_{i \in I_{2}} \int_{0}^{\Delta t} \int_{\sigma_{i}^{-} t}^{\sigma_{i}^{+} t} M(t, x) d x d t=: \sum_{k=1}^{4} J_{k} .
\end{gathered}
$$

In a region $\left\{(x, t): \sigma_{i}^{+} t \leqslant x \leqslant \sigma_{i+1}^{-} t\right\}$ where $W_{C} \equiv U_{i}$ and $I_{C}=I_{i}(\omega, \nu)$ are constant states, one has after (40) and (41)

$$
W_{G}(t, x)=U_{i}+\operatorname{tg}\left(I_{C}, U_{i}\right),
$$

and

$$
I_{G}(t, x, \omega, \nu)=I_{i}(\omega, \nu)+\operatorname{ct} \mathcal{S}\left(I_{C}, U_{i}\right)
$$

then for $i=1,2$

$$
\partial_{t} W_{G}+\partial_{x} f\left(W_{G}\right)-g\left(I_{G}, W_{G}\right)=g\left(I_{C}, U_{i}\right)-g\left(I_{G}, W_{G}\right) .
$$

Multiplying by $\phi$ and integrating by parts we find

$$
\int_{0}^{\Delta t} \int_{\sigma_{i}^{+} t}^{\sigma_{i+1}^{-} t} M(t, x) d x d t=\int_{\sigma_{i}^{+} \Delta t}^{\sigma_{i+1}^{-} \Delta t} \phi(\Delta t, x) W_{G}(\Delta t, x) d x
$$




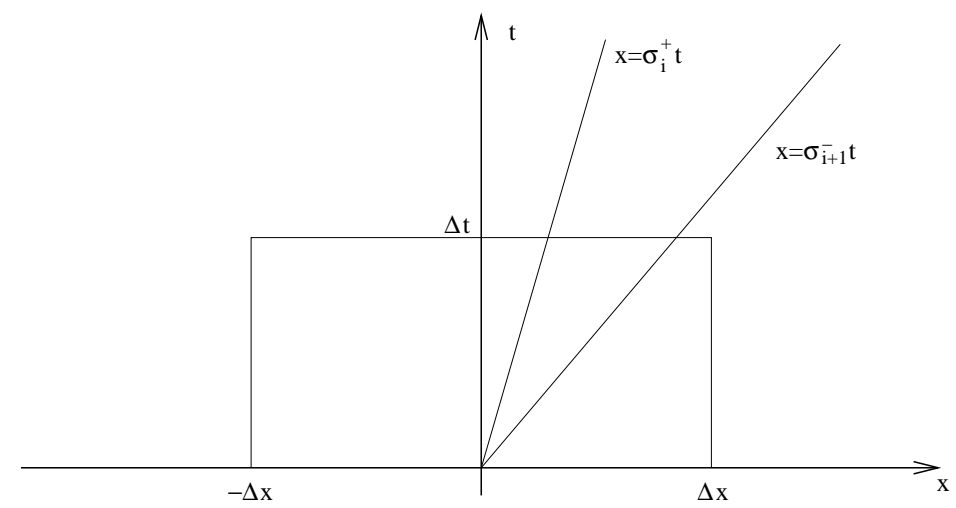

Figure 1: Representation of a part of the Riemann solution. Note that due to CFL condition (42), lines $x=\sigma_{i}^{ \pm} t$ never intersect the vertical boundary of the cell $[-\Delta x, \Delta x] \times[0, \Delta t]$.

$$
\begin{gathered}
+\int_{0}^{\Delta t}\left\{f \left(W_{G}\left(\Delta t, \sigma_{i+1}^{-} \Delta t\right) \phi\left(\Delta t, \sigma_{i+1}^{-} \Delta t\right)-f\left(W_{G}\left(\Delta t, \sigma_{i}^{+} \Delta t\right) \phi\left(\Delta t, \sigma_{i}^{+} \Delta t\right)\right\} d t\right.\right. \\
-\int_{0}^{\Delta t}\left\{\sigma_{i+1}^{-} \phi\left(t, \sigma_{i+1}^{-} t\right) W_{G}\left(t, \sigma_{i+1}^{-} t\right)-\sigma_{i}^{+} \phi\left(t, \sigma_{i}^{+} t\right) W_{G}\left(t, \sigma_{i}^{+} t\right)\right\} d t \\
\quad-\int_{0}^{\Delta t} \int_{\sigma_{i}^{+} t}^{\sigma_{i+1}^{-} t}\left(g\left(I_{C}(t), U_{i}\right)-g\left(I_{G}, W_{G}\right)\right) \phi(t, x) d x d t .
\end{gathered}
$$

Applying Lemma 1, we have

$$
\begin{aligned}
\left|g\left(I_{C}, U_{i}\right)-g\left(I_{G}, W_{G}\right)\right| \leqslant & h_{2}\left(\left|U_{i}\right|,\left|W_{G}\right|,\left\|I_{C}\right\|_{L_{\omega, \nu}^{1}\left([-1,1] \times \mathbb{R}^{+}\right)},\left\|I_{G}\right\|_{L_{\omega, \nu}^{1}\left([-1,1] \times \mathbb{R}^{+}\right)}\right) \\
& \times\left[t\left|g\left(I_{C}, U_{i}\right)\right|+c t\left\|\mathcal{S}\left(I_{C}, U_{i}\right)\right\|_{L_{\omega, \nu}^{1}\left([-1,1] \times \mathbb{R}^{+}\right)}\right] \\
\leqslant & t h_{2}\left(\left|U_{i}\right|,\left|W_{G}\right|,\left\|I_{C}\right\|_{L_{\omega, \nu}^{1}\left([-1,1] \times \mathbb{R}^{+}\right)},\left\|I_{G}\right\|_{L_{\omega, \nu}^{1}\left([-1,1] \times \mathbb{R}^{+}\right)}\right) \\
& \times\left[h_{1}\left(\left|U_{i}\right|,\left\|I_{C}\right\|_{L_{\omega, \nu}^{1}\left([-1,1] \times \mathbb{R}^{+}\right)}\right)+c h_{0}\left(\left|U_{i}\right|,\left\|I_{C}\right\|_{L_{\omega, \nu}^{1}\left([-1,1] \times \mathbb{R}^{+}\right)}\right)\right] \\
\leqslant & C t,
\end{aligned}
$$

where $C$ depends only on $\mathcal{U}, h_{0}, h_{1}, h_{2}$. Hence, since $\sigma_{i}^{+}$and $\sigma_{i}^{-}$are bounded, according to (42),

$$
\left|\int_{0}^{\Delta t} \int_{\sigma_{i}^{+} t}^{\sigma_{i+1}^{-} t}\left(g\left(I_{C}, U_{i}\right)-g\left(I_{G}, W_{G}\right)\right) \phi(t, x) d x d t\right| \leqslant C(\Delta t)^{3}\|\phi\|_{C^{0}},
$$

where $C$ depends only on $g$ and $\mathcal{U}$. Thus, we finally get

$$
\begin{gathered}
\int_{0}^{\Delta t} \int_{\sigma_{i}^{+} t}^{\sigma_{i+1}^{-} t} M(t, x) d x d t=\int_{\sigma_{i}^{+} \Delta t}^{\sigma_{i+1}^{-} \Delta t} \phi(\Delta t, x) W_{G}(\Delta t, x) d x \\
+\int_{0}^{\Delta t}\left\{f \left(W_{G}\left(\Delta t, \sigma_{i+1}^{-} \Delta t\right) \phi\left(\Delta t, \sigma_{i+1}^{-} \Delta t\right)-f\left(W_{G}\left(\Delta t, \sigma_{i}^{+} \Delta t\right) \phi\left(\Delta t, \sigma_{i}^{+} \Delta t\right)\right\} d t\right.\right. \\
-\int_{0}^{\Delta t}\left\{\sigma_{i+1}^{-} \phi\left(t, \sigma_{i+1}^{-} t\right) W_{G}\left(t, \sigma_{i+1}^{-} t\right)-\sigma_{i}^{+} \phi\left(t, \sigma_{i}^{+} t\right) W_{G}\left(t, \sigma_{i}^{+} t\right)\right\} d t \\
+O\left((\Delta t)^{3}\right)\|\phi\|_{X} .
\end{gathered}
$$

In the same stroke

$$
J_{2}=\int_{0}^{\Delta t} \int_{-\Delta x}^{\sigma_{1}^{-} t} M(t, x) d x d t
$$




$$
\begin{gathered}
=\int_{-\Delta x}^{\sigma_{1}^{-} \Delta t} \phi(\Delta t, x) W_{G}(\Delta t, x) d x-\int_{-\Delta x}^{0} \phi(0, x) W_{G}(0, x) d x \\
+\int_{0}^{\Delta t}\left\{f \left(W_{G}\left(t, \sigma_{1}^{-} t\right) \phi\left(t, \sigma_{1}^{-} t\right)-f\left(W_{G}(t,-\Delta x) \phi(t,-\Delta x)\right\} d t\right.\right. \\
\quad-\int_{0}^{\Delta t} \sigma_{1}^{-} \phi\left(t, \sigma_{1}^{-} t\right) W_{G}\left(t, \sigma_{1}^{-} t\right) d t \\
-\int_{0}^{\Delta t} \int_{-\Delta x}^{\sigma_{1}^{-} t}\left(g\left(I_{C}, U_{i}\right)-g\left(I_{G}, W_{G}\right)\right) \phi(t, x) d x d t .
\end{gathered}
$$

Using Lemma 1 here again, the last two terms are bounded by $O\left((\Delta t)^{3}+(\Delta t)^{2} \Delta x\right)\|\phi\|_{X}$, and we get

$$
\begin{gathered}
\int_{0}^{\Delta t} \int_{-\Delta x}^{\sigma_{1}^{-} t} M(t, x) d x d t \\
=\int_{-\Delta x}^{\sigma_{1}^{-} \Delta t} \phi(\Delta t, x) W_{G}(\Delta t, x) d x-\int_{-\Delta x}^{0} \phi(0, x) W_{G}(0, x) d x \\
+\int_{0}^{\Delta t}\left\{f \left(W_{G}\left(t, \sigma_{1}^{-} t\right) \phi\left(t, \sigma_{1}^{-} t\right)-f\left(W_{G}(t,-\Delta x) \phi(t,-\Delta x)\right\} d t\right.\right. \\
-\int_{0}^{\Delta t} \sigma_{1}^{-} \phi\left(t, \sigma_{1}^{-} t\right) W_{G}\left(t, \sigma_{1}^{-} t\right) d t+O\left((\Delta t)^{3}+(\Delta t)^{2} \Delta x\right)\|\phi\|_{X} .
\end{gathered}
$$

Finally

$$
\begin{gathered}
J_{3}=\int_{0}^{\Delta t} \int_{\sigma_{3}^{+} t}^{\Delta x} M(t, x) d x d t=\int_{\sigma_{3}^{+} \Delta t}^{\Delta x} \phi(\Delta t, x) W_{G}(\Delta t, x) d x-\int_{0}^{\Delta x} \phi(0, x) W_{G}(0, x) d x \\
+\int_{0}^{\Delta t}\left\{f \left(W_{G}(t, \Delta x) \phi(t, \Delta x)-f\left(W_{G}\left(t, \sigma_{3}^{+} t\right) \phi\left(t, \sigma_{3}^{+} t\right)\right\} d t\right.\right. \\
\quad-\int_{0}^{\Delta t} \sigma_{3}^{+} \phi\left(t, \sigma_{3}^{+} t\right) W_{G}\left(t, \sigma_{3}^{+} t\right) d t \\
-\int_{0}^{\Delta t} \int_{\sigma_{3}^{+} t}^{\Delta x}\left(g\left(I_{C}, U_{i}\right)-g\left(I_{G}, W_{G}\right)\right) \phi(t, x) d x d t
\end{gathered}
$$

and, still with the help of Lemma 1, the last two terms are bounded by $O\left((\Delta t)^{3}+(\Delta t)^{2} \Delta x\right)\|\phi\|_{X}$, so

$$
\begin{gathered}
\int_{0}^{\Delta t} \int_{\sigma_{3}^{+} t}^{\Delta x} M(t, x) d x d t=\int_{\sigma_{3}^{+} \Delta t}^{\Delta x} \phi(\Delta t, x) W_{G}(\Delta t, x) d x-\int_{0}^{\Delta x} \phi(0, x) W_{G}(0, x) d x \\
\quad+\int_{0}^{\Delta t}\left\{f \left(W_{G}(t, \Delta x) \phi(t, \Delta x)-f\left(W_{G}\left(t, \sigma_{3}^{+} t\right) \phi\left(t, \sigma_{3}^{+} t\right)\right\} d t\right.\right. \\
-\int_{0}^{\Delta t} \sigma_{3}^{+} \phi\left(t, \sigma_{3}^{+} t\right) W_{G}\left(t, \sigma_{3}^{+} t\right) d t+O\left((\Delta t)^{3}+(\Delta t)^{2} \Delta x\right)\|\phi\|_{X} .
\end{gathered}
$$

Next, we assume that $W_{C}(t, x)$ consists in a $i$-rarefaction wave in the fan region $\left\{(x, t): \sigma_{i}^{-} t<x<\sigma_{i}^{+} t\right\}$, so that $W_{G}$ rewrites

$$
W_{G}(t, x)=\tilde{W}_{C}(\zeta)+\operatorname{tg}\left(I_{C}(t), W_{C}(t)\right),
$$

where $\zeta=\frac{x}{t}$ and $\tilde{W}_{C}$ is the $i$-rarefaction wave. Following [12] we have

$$
\partial_{t} W_{G}+\partial_{x}\left(f\left(W_{G}\right)\right)-g\left(I_{G}, W_{G}\right)=\frac{1}{t}\left(D f\left(W_{G}\right)-\frac{x}{t}\right) \tilde{W}_{C}^{\prime}\left(\frac{x}{t}\right)+g\left(I_{C}, W_{C}\right)-g\left(I_{G}, W_{G}\right) .
$$


Hence, using the fact that $\left(D f\left(\tilde{W}_{C}(\xi)\right)-\xi\right) \tilde{W}_{C}^{\prime}(\xi)=0$,

$$
\partial_{t} W_{G}+\partial_{x}\left(f\left(W_{G}\right)\right)-g\left(I_{G}, W_{G}\right)=\left(D f\left(W_{G}\right)-D f\left(W_{C}\right)\right) \partial_{x} W_{C}+g\left(I_{C}, W_{C}\right)-g\left(I_{G}, W_{G}\right) .
$$

All terms in the right-hand side of the above equality are dealt with exactly as before, except for the first one, for which we use the fact that $\|f\|_{C^{2}(\mathcal{U})}$ is bounded, and

$$
\begin{aligned}
\left|\left(D f\left(W_{G}\right)-D f\left(W_{C}\right)\right) \partial_{x} W_{C}\right| & \leqslant\|f\|_{C^{2}(\mathcal{U})} \Delta t\left|g\left(I_{C}, W_{C}\right)\right|\left|\partial_{x} W_{C}\right| \\
& \leqslant C \Delta t\left|\partial_{x} W_{C}\right|
\end{aligned}
$$

where $C$ depends only on $f, g$, and $\mathcal{U}$. Hence,

$$
\left|\int_{0}^{\Delta t} \int_{\sigma_{i}^{+} \Delta t}^{\sigma_{i}^{-} \Delta t}\left(D f\left(W_{G}\right)-D f\left(W_{C}\right)\right) \partial_{x} W_{C} \phi\right| \leqslant C \Delta t \int_{0}^{\Delta t} T V\left(W_{C}\right) d t\|\phi\|_{X} .
$$

For a rarefaction wave, the total variation is non-increasing, so the above term is bounded by $O\left(\Delta t^{2} \mid U_{R}-\right.$ $\left.U_{L} \mid\right)\|\phi\|_{X}$. Hence, we have

$$
\begin{gathered}
\int_{0}^{\Delta t} \int_{\sigma_{i}^{-} t}^{\sigma_{i}^{+} t} M(t, x) d x d t=\int_{\sigma_{i}^{+} \Delta t}^{\sigma_{i}^{-} \Delta t} \phi(\Delta t, x) W_{G}(\Delta t, x) d x \\
-\int_{0}^{\Delta t}\left\{f \left(W_{G}\left(t, \sigma_{i}^{-} t\right) \phi\left(t, \sigma_{i}^{-} t\right)-f\left(W_{G}\left(t, \sigma_{i}^{+} t\right) \phi\left(t, \sigma_{i}^{+} t\right)\right\} d t\right.\right. \\
+\int_{0}^{\Delta t}\left\{\sigma_{i}^{-} \phi\left(t, \sigma_{i+1}^{-} t\right) W_{G}\left(t, \sigma_{i}^{-} t\right)-\sigma_{i}^{+} \phi\left(t, \sigma_{i}^{+} t\right) W_{G}\left(t, \sigma_{i}^{+} t\right)\right\} d t \\
+O\left(\Delta t^{2}\left(\Delta t+\left|U_{R}-U_{L}\right|\right)\right)\|\phi\|_{X} .
\end{gathered}
$$

Collecting all the previous estimates (45), (46), (47) and (48) proves (43).

Defining in the same spirit the auxiliary function $N(t, x):=I_{G} \partial_{t} \psi+c \omega I_{G} \partial_{x} \psi+c \mathcal{S}\left(I_{G}, W_{G}\right) \psi$, we have the following decomposition of $\int N d x d t$ on the elementary cell $[0, \Delta t] \times[-\Delta x, \Delta x]$

$$
\int_{0}^{\Delta t} \int_{-\Delta x}^{\Delta x} N(t, x) d x d t=\int_{0}^{\Delta t} \int_{-\Delta x}^{\sigma t} M(t, x) d x d t+\int_{0}^{\Delta t} \int_{\sigma t}^{\Delta x} M(t, x) d x d t
$$

where $\sigma=c \omega$.

As $I_{G}(t, x, \omega, \nu)=I_{C}(\omega, \nu)+c t \mathcal{S}\left(I_{C}, W_{C}\right)$, we get

$$
\partial_{t} I_{G}+c \omega \partial_{x} I_{G}-c \mathcal{S}\left(I_{G}, W_{G}\right)=c \mathcal{S}\left(I_{C}, U_{i}\right)-c \mathcal{S}\left(I_{G}, W_{G}\right) .
$$

Multiplying by $\psi$ and integrating by parts we find

$$
\begin{gathered}
\int_{0}^{\Delta t} \int_{-\Delta x}^{\sigma t} N(t, x) d x d t \\
=\int_{-\Delta x}^{\sigma \Delta t} \psi(\Delta t, x) I_{G}(\Delta t, x) d x-\int_{-\Delta x}^{0} \psi(0, x) I_{G}(0, x) d x \\
+\int_{0}^{\Delta t} \sigma\left\{I_{G}(t, \sigma t) \psi(t, \sigma t)-I_{G}(t,-\Delta x) \psi(t,-\Delta x)\right\} d t \\
-c \int_{0}^{\Delta t} \int_{-\Delta x}^{\sigma t}\left(\mathcal{S}\left(I_{C}, U_{i}\right)-\mathcal{S}\left(I_{G}, W_{G}\right)\right) \psi(t, x) d x d t .
\end{gathered}
$$

Using the previous arguments the last two terms are bounded by $O\left((\Delta t)^{3}+(\Delta t)^{2}\left|I_{R}-I_{L}\right|\right)\|\psi\|_{Y}$, and we get

$$
\int_{0}^{\Delta t} \int_{-\Delta x}^{\sigma t} N(t, x) d x d t
$$




$$
\begin{gathered}
=\int_{-\Delta x}^{\sigma \Delta t} \psi(\Delta t, x) I_{G}(\Delta t, x) d x-\int_{-\Delta x}^{0} \psi(0, x) I_{G}(0, x) d x \\
\quad+\int_{0}^{\Delta t} \sigma\left\{I_{G}(t, \sigma t) \psi(t, \sigma t)-I_{G}(t,-\Delta x) \psi(t,-\Delta x)\right\} d t \\
-\int_{0}^{\Delta t} \sigma \phi(t, \sigma t) W_{G}(t, \sigma t) d t+O\left((\Delta t)^{3}+(\Delta t)^{2} \Delta x\right)\|\psi\|_{Y} .
\end{gathered}
$$

Finally

$$
\begin{gathered}
\int_{0}^{\Delta t} \int_{\sigma t}^{\Delta x} N(t, x) d x d t=\int_{\sigma \Delta t}^{\Delta x} \psi(\Delta t, x) I_{G}(\Delta t, x) d x-\int_{0}^{\Delta x} \psi(0, x) I_{G}(0, x) d x \\
+\int_{0}^{\Delta t} \sigma\left\{I_{G}(t, \Delta x) \psi(t, \Delta x)-I_{G}(t, \sigma t) \psi(t, \sigma t)\right\} d t \\
\quad-\int_{0}^{\Delta t} \int_{\sigma t}^{\Delta x}\left(\mathcal{S}\left(I_{C}, U_{i}\right)-\mathcal{S}\left(I_{G}, W_{G}\right)\right) \psi(t, x) d x d t
\end{gathered}
$$

and

$$
\left|\int_{0}^{\Delta t} \int_{\sigma t}^{\Delta x}\left(\mathcal{S}\left(I_{C}, U_{i}\right)-\mathcal{S}\left(I_{G}, W_{G}\right)\right) \psi(t, x) d x d t\right|=O\left((\Delta t)^{3}+(\Delta t)^{2}\left|I_{R}-I_{L}\right|\right)\|\psi\|_{X},
$$

SO

$$
\begin{gathered}
\int_{0}^{\Delta t} \int_{\sigma t}^{\Delta x} N(t, x) d x d t=\int_{\sigma \Delta t}^{\Delta x} \psi(\Delta t, x) I_{G}(\Delta t, x) d x-\int_{0}^{\Delta x} \psi(0, x) I_{G}(0, x) d x \\
\quad+\int_{0}^{\Delta t} \sigma\left\{I_{G}(t, \Delta x) \psi(t, \Delta x)-I_{G}(t, \sigma t) \psi(t, \sigma t)\right\} d t \\
\quad-\int_{0}^{\Delta t} \sigma \phi(t, \sigma t) W_{G}(t, \sigma t) d t+O\left((\Delta t)^{3}+(\Delta t)^{2}\left|I_{R}-I_{L}\right|\right)\|\psi\|_{Y} .
\end{gathered}
$$

Collecting estimates (49) and (50) proves (44)

\section{Interaction of waves}

Following [9] (see also [24] for a complete presentation) we define the wave interaction potential involving two solutions of the classical Riemann problem of respective strengths $\alpha:=\left(\alpha_{1}, \alpha_{2}, \alpha_{3}, \alpha_{4}\right)$ and $\beta:=\left(\beta_{1}, \beta_{2}, \beta_{3}, \beta_{4}\right)$, by

$$
D(\alpha, \beta):=\sum_{i>j}\left|\alpha_{i}\right|\left|\beta_{j}\right|+\sum_{k} Q_{k}\left(\alpha_{k}, \beta_{k}\right),
$$

where

$$
Q_{i}\left(\alpha_{i}, \beta_{i}\right)=\left|\alpha_{i}\right|\left|\beta_{i}\right|-\alpha_{i}^{+} \beta_{i}^{+},
$$

with $z^{+}=\sup (z, 0)$, if the $i-$ field is genuinely non linear, and

$$
Q_{i}\left(\alpha_{i}, \beta_{i}\right)=0,
$$

if the $i$-field is linearly degenerate.

The following result due to Glimm [9] describes wave interaction estimates

Proposition 3 (Glimm). Let $U_{L}, U_{M}, U_{R}$ be three constant states in $\mathcal{U}$ and let $(\alpha, \beta, \gamma)$ the strengths of the solutions of the Riemann problems $C R U\left(x_{0}, t_{0} ; U_{L}, U_{M}\right), C R U\left(x_{0}, t_{0} ; U_{M}, U_{R}\right)$ and $C R U\left(x_{0}, t_{0} ; U_{L}, U_{R}\right)$.

The following properties hold

1.

$$
|\gamma-\alpha-\beta|=O(1) D(\alpha, \beta) .
$$


2. If $V_{L}, V_{R}$ are two other constant states in $\mathcal{U}$ and $\delta$ is the strength of the solution of the Riemann problem $C R U\left(x_{0}, t_{0} ; V_{L}, V_{M}\right)$

$$
D(\gamma, \delta)=D(\alpha, \delta)+D(\beta, \delta)+O(1)|\delta| D(\alpha, \beta)
$$

3. The mapping $\left(U_{L}, U_{R}, t_{0}, x_{0}\right) \rightarrow \alpha: \mathcal{U} \times \mathcal{U} \times \mathbb{R}_{+} \times \mathbb{R} \rightarrow \mathbb{R}^{3}$ is a $C^{2}$ function of its arguments. Moreover for $\left(U_{L}, U_{R}\right)$ and $\left(U_{L}^{\prime}, U_{R}^{\prime}\right)$ in $\mathcal{U} \times \mathcal{U}$ and $\left(t_{0}, x_{0}\right)$ and $\left(t_{0}^{\prime}, x_{0}^{\prime}\right)$ in $\mathbb{R}_{+} \times \mathbb{R}$, one has

$$
\left|\alpha^{\prime}-\alpha\right|=O(1)\left\{|\alpha|\left(\left|U_{L}^{\prime}-U_{L}\right|+\left|U_{R}^{\prime}-U_{R}\right|\right)+\left(\left|U_{R}^{\prime}-U_{L}^{\prime}-\left(U_{R}-U_{L}\right)\right|\right)\right\},
$$

where $\alpha=\alpha\left(U_{L}, U_{R} ; t_{0}, x_{0}\right)$ and $\alpha^{\prime}=\alpha\left(U_{L}^{\prime}, U_{R}^{\prime} ; t_{0}^{\prime}, x_{0}^{\prime}\right)$.

4. For $\left(U_{L}, U_{R}\right),\left(V_{L}, V_{R}\right),\left(U_{l}^{\prime}, U_{R}^{\prime}\right)$ and $\left(V_{L}^{\prime}, V_{R}^{\prime}\right)$ in $\mathcal{U} \times \mathcal{U}$ and for $\left(t_{1}, x_{1}\right),\left(t_{2}, x_{2}\right),\left(t_{1}^{\prime}, x_{1}^{\prime}\right)$, and $\left(t_{2}^{\prime}, x_{2}^{\prime}\right)$ in $\mathbb{R}_{+} \times \mathbb{R}$, one has

$$
\begin{gathered}
D\left(\alpha^{\prime}, \beta^{\prime}\right)=D(\alpha, \beta)+O(1)|\alpha|\left|\left(V_{R}^{\prime}-V_{L}^{\prime}\right)+\left(V_{R}-V_{L}\right)\right|+O(1)|\beta|\left|\left(U_{R}^{\prime}-U_{L}^{\prime}\right)+\left(U_{R}-U_{L}\right)\right| \\
+O(1)|\alpha||\beta|\left(\left|U_{L}^{\prime}-U_{L}\right|+\left|U_{R}^{\prime}-U_{R}\right|+\left|V_{L}^{\prime}-V_{L}\right|+\left|V_{R}^{\prime}-V_{R}\right|\right) \\
\quad+O(1)\left|U_{R}^{\prime}-U_{L}^{\prime}-\left(U_{R}-U_{L}\right)\right| \cdot\left|V_{R}^{\prime}-V_{L}^{\prime}-\left(V_{R}-V_{L}\right)\right|,
\end{gathered}
$$

where $\alpha=\alpha\left(U_{L}, U_{R} ; t_{1}, x_{1}\right), \alpha^{\prime}=\alpha\left(U_{L}^{\prime}, U_{R}^{\prime} ; t_{1}^{\prime}, x_{1}^{\prime}\right), \beta=\beta\left(V_{L}, V_{R} ; t_{2}, x_{2}\right)$ and $\beta^{\prime}=\beta\left(V_{L}^{\prime}, V_{R}^{\prime} ; t_{2}^{\prime}, x_{2}^{\prime}\right)$.

5. Let $U_{L}, U_{L}+u_{L}, U_{R}, U_{R}+u_{R}$ and $U_{M}$ be constant states in $\mathcal{U}$ and let $(\alpha, \beta, \gamma)$ the strengths of the solutions of the Riemann problems $C R U\left(x_{0}-\Delta x, t_{0} ; U_{L}, U_{M}\right), C R U\left(x_{0}+\Delta x, t_{0} ; U_{M}, U_{R}\right)$ and $C R U\left(x_{0}, t_{0}+\Delta t ; U_{L}+\right.$ $\left.u_{L}, U_{R}+u_{R}\right)$. We have

$$
|\gamma|=|\alpha|+|\beta|+O(1) D(\alpha, \beta)+O(1)(|\alpha|+|\beta|)\left(\left|u_{L}\right|+\left|u_{R}\right|\right)+O(1)\left(\left|u_{R}-u_{L}\right|\right) .
$$

6. For the previous $\alpha, \beta$ and $\gamma$, for $\left(V_{L}, V_{R}\right)$ in $\mathcal{U} \times \mathcal{U}$ and for $\left(t_{1}, x_{1}\right)$ in $\mathbb{R}_{+} \times \mathbb{R}$, one has

$$
D(\gamma, \delta)=D(\alpha, \delta)+D(\beta, \delta)+O(1)|\delta| D(\alpha, \beta)+O(1)|\delta|\left|u_{R}-u_{L}\right|+O(1)|\delta|(|\alpha|+|\beta|)\left(\left|u_{L}\right|+\left|u_{R}\right|\right)
$$

The first four items of the previous result were proved by Glimm [9] for genuinely non linear fields and extended by Tai Ping Liu [24] for linearly degenerate fields. The two last items were proved by Hong and LeFloch [12] in a more general context (in [12], the right-hand side $f$ depends on $x$ and $t$ ).

Remark 2. Let $I_{L}, I_{M}, I_{R}$ be three constant (radiative) states. The strengths of the solutions of the Riemann problems $C R I\left(x_{0}, t_{0} ; I_{L}, I_{M}\right), C R I\left(x_{0}, t_{0} ; I_{M}, I_{R}\right)$ and $C R I\left(x_{0}, t_{0} ; I_{L}, I_{R}\right)$ satisfy (non interacting framework)

$$
\gamma_{r}=\alpha_{r}+\beta_{r}
$$

Then we have the following consequence of Proposition 3

Proposition 4. Let us denote by $u_{L}, u_{R}$ the numbers

$$
u_{L}:=-\Delta t g\left(I_{L}, U_{L}\right) \text { for } x<0,
$$

and

$$
u_{R}:=-\Delta t g\left(I_{R}, U_{R}\right) \text { for } x>0 .
$$

The following properties hold $\left(\alpha, \beta\right.$ and $\gamma$ are defined in Proposition 3, item 5, and $\overline{\sigma_{a}}$, $\overline{\sigma_{s}}$ are defined in Lemma 1)

$$
\begin{aligned}
|\gamma|=|\alpha|+|\beta|+O(1) D(\alpha, \beta) & \\
+O(1)(|\alpha|+|\beta|)\left(\overline{\sigma_{a}}\left(t_{0}\right)+\right. & \left.\overline{\sigma_{s}}\left(t_{0}\right)\right) \Delta t\left(\left|U_{L}\right|+\left|U_{R}\right|+\left\|I_{L}\right\|_{L_{\omega, \nu}^{1}\left([-1,1] \times \mathbb{R}^{+}\right)}\left\|I_{R}\right\|_{L_{\omega, \nu}^{1}\left([-1,1] \times \mathbb{R}^{+}\right)}\right) \\
& +O(1)\left(\overline{\sigma_{a}}\left(t_{0}\right)+\overline{\sigma_{s}}\left(t_{0}\right)\right) \Delta t\left(\left|U_{L}-U_{R}\right|+\left\|I_{L}-I_{R}\right\|_{L_{\omega, \nu}^{1}\left([-1,1] \times \mathbb{R}^{+}\right)}\right)
\end{aligned}
$$


and

$$
\begin{aligned}
D(\gamma, \delta)=D(\alpha, \delta)+D(\beta, \delta)+O(1)|\delta| D(\alpha, \beta) & \\
+O(1)|\delta|(|\alpha|+|\beta|)\left(\overline{\sigma_{a}}\left(t_{0}\right)\right. & \left.+\overline{\sigma_{s}}\left(t_{0}\right)\right) \Delta t\left(\left|U_{L}\right|+\left|U_{R}\right|+\left\|I_{L}\right\|_{L_{\omega, \nu}^{1}\left([-1,1] \times \mathbb{R}^{+}\right)}\left\|I_{R}\right\|_{L_{\omega, \nu}^{1}\left([-1,1] \times \mathbb{R}^{+}\right)}\right) \\
& +O(1)\left(\overline{\sigma_{a}}\left(t_{0}\right)+\overline{\sigma_{s}}\left(t_{0}\right)\right) \Delta t\left(\left|U_{L}-U_{R}\right|+\left\|I_{L}-I_{R}\right\|_{L_{\omega, \nu}^{1}\left([-1,1] \times \mathbb{R}^{+}\right)}\right) .
\end{aligned}
$$

Proof: Applying (56), we get first

$$
|\gamma|=|\alpha|+|\beta|+O(1)(|\alpha|+|\beta|)\left(\left|u_{L}\right|+\left|u_{R}\right|\right)+O(1)\left|u_{L}-u_{R}\right| .
$$

Now let us estimate the terms $\left|u_{L}\right|+\left|u_{R}\right|$. For this purpose, we apply (26) of Lemma 1 . This gives

$$
\left.\left|u_{L}\right| \leqslant \Delta t\left|g\left(I_{L}, U_{L}\right)\right| \leqslant \Delta t h_{1}\left(\mid U_{L}\right) \mid,\left\|I_{L}\right\|_{L_{\omega, \nu}^{1}\left(\mathbb{R}^{+} \times[-1,1]\right)}\right)\left(\overline{\sigma_{a}}\left(t_{0}\right)+\overline{\sigma_{s}}\left(t_{0}\right)\right) .
$$

Simlarly,

$$
\left.\left|u_{R}\right| \leqslant \Delta t h_{1}\left(\mid U_{R}\right) \mid,\left\|I_{R}\right\|_{L_{\omega, \nu}^{1}\left(\mathbb{R}^{+} \times[-1,1]\right)}\right)\left(\overline{\sigma_{a}}\left(t_{0}\right)+\overline{\sigma_{s}}\left(t_{0}\right)\right) .
$$

Next, we bound the term $\left|u_{L}-u_{R}\right|$. For this purpose, we apply (27) of Lemma 1, finding

$$
\begin{aligned}
\left|u_{L}-u_{R}\right|=\Delta t\left|g\left(I_{L}, U_{L}\right)-g\left(I_{R}, U_{R}\right)\right| \leqslant h_{2} & \left(\left|U_{L}\right|,\left|U_{R}\right|,\left\|I_{L}\right\|_{L_{\omega, \nu}^{1}\left([-1,1] \times \mathbb{R}^{+}\right)},\left\|I_{R}\right\|_{L_{\omega, \nu}^{1}\left([-1,1] \times \mathbb{R}^{+}\right)}\right) \\
& \times\left[\left|U_{L}-U_{R}\right|+\left\|I_{L}-I_{R}\right\|_{L_{\omega, \nu}^{1}\left([-1,1] \times \mathbb{R}^{+}\right)}\right]\left(\overline{\sigma_{a}}(t)+\overline{\sigma_{s}}(t)\right) .
\end{aligned}
$$

Inserting estimates (64), (65) and (66) into (63), we find (61). Estimate (62) is obtained in the same way.

\section{The generalized Glimm's scheme}

We approximate the problem (15)(16)(17) by using a two-step scheme relying on the Glimm's method as follows.

Let $\mathcal{U}$ be a small neighborhood of $\left(\rho_{\infty}, v_{\infty}, S_{\infty}, I_{\infty}\right)$ in $\mathbb{R}^{4}$ (see Definition 1 ). Given space and time steps $\Delta t$ and $\Delta x$ satisfying the mixed CFL condition

$$
\frac{\Delta t}{\Delta x} \max \left\{c, \max _{i=1,2,3}\left(\sup _{u \in \mathcal{U}}\left|\lambda_{i}(u)\right|\right)\right\} \leqslant 1
$$

and a sequence $a \equiv\left(a_{\ell}\right)_{\ell \geqslant 0}$ of real numbers equidistributed in the interval $(-1,1)$, let us define (see $\left.[9,22]\right)$ the discretization of the $(t, x)$ half-plane by the mesh points

$$
A_{n}^{\ell}=\left(t_{\ell}, x_{n}\right) \equiv(\ell \Delta t, n \Delta x) \text { for } \ell \in \mathbb{N}, n \in \mathbb{Z}, n+\ell \text { odd. }
$$

Connecting nearest-neighbor mesh points by segments defines a partition of the plane into diamond-like regions.

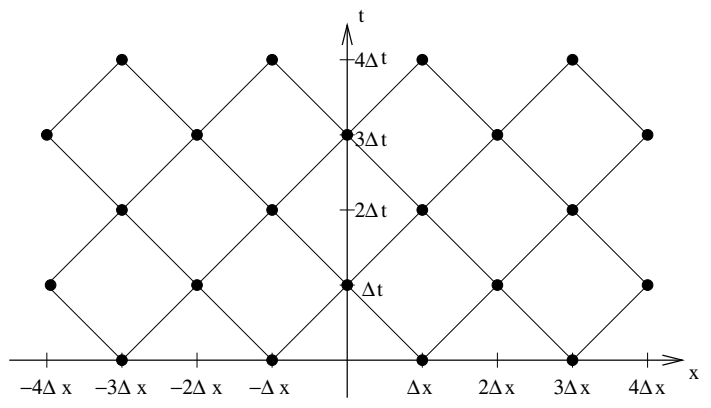

Figure 2: The mesh points defined by (68). 


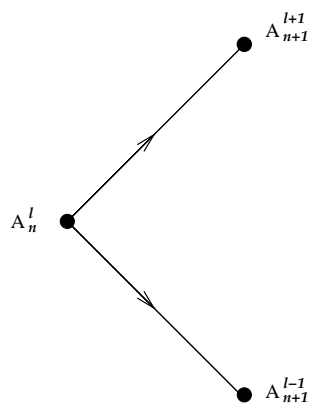

Figure 3: Given a point in a mesh curve, the next one should be one of its two nearest right neighbors

Definition 2. Consider a mesh $\left(A_{n}^{\ell}\right)_{\ell \in \mathbb{N}, n \in \mathbb{Z}}$ as defined by (68). We call mesh curve $L$ an unbounded piecewise linear curve lying on diamond boundaries of $\left(A_{n}^{\ell}\right)_{\ell \in \mathbb{N}, n \in \mathbb{Z}}$, which satisfies the following: for any point $A_{n}^{\ell} \in L$, the next point is either $A_{n+1}^{\ell-1}$ or $A_{n+1}^{\ell+1}$ (see figure 3).

Any mesh curve $L$ divides the half-plane $t \geqslant 0$ into two components $L^{+}$and $L$, where $L^{-}$contains the axis $t=0$. We define an order on the mesh curves: $L_{1}>L_{2}$ if any point of $L_{1}$ either is on $L_{2}$ or is contained in $L_{2}^{+}$. We say that $L_{1}$ is an immediate successor of $L_{2}$ if $L_{1}>L_{2}$ and if each mesh point of $L_{1}$ except one is on $L_{2}$.

Let us approximate the initial data (16) by

$$
U_{\Delta x}(x, 0)=U_{0}(n \Delta x), \text { for } x \in[(n-1) \Delta x,(n+1) \Delta x], \quad n \text { odd, }
$$

which allows us to construct for $0<t \leqslant \Delta t$ a solution $W_{C}$ of each classical Riemann problem with initial data $U_{\Delta x}$ and, due to the CFL condition, to define the solution of the Cauchy problem (38) in the strip $\{(x, t) \in \mathbb{R} \times] 0, \Delta t[\}$.

Approximating as well the initial data (17) by

$$
I_{\Delta x}(x, 0, \omega, \nu)=I_{0}(n \Delta x, \omega, \nu) \text { for } x \in[(n-1) \Delta x,(n+1) \Delta x], \quad n \text { odd },
$$

allows us to construct for $0<t \leqslant \Delta t$ a solution $I_{C}$ of each classical Riemann problem with initial data $I_{\Delta x}$ and, due to the CFL condition, to define the solution of the Cauchy problem (39) in the strip $\{(x, t) \in \mathbb{R} \times] 0, \Delta t[\}$.

Using then (40) and (41) we can construct for $0<t \leqslant \Delta t$ the approximate solutions $W_{G}$ and $I_{G}$ for each generalized Riemann problem (37) with the previous initial data $U_{\Delta x}$ and $I_{\Delta x}$, defining the solution $\left(I_{\Delta x}(x, t, \omega, \nu), U_{\Delta x}(x, t)\right)$ in the strip $\{(x, t) \in \mathbb{R} \times] 0, \Delta t[\}$.

Supposing now $\left(I_{\Delta x}(x, t, \omega, \nu), U_{\Delta x}(x, t)\right)$ well defined in the strip $\{(x, t) \in \mathbb{R} \times[(\ell-1) \Delta t, \ell \Delta t[\}$, we set

$$
\begin{aligned}
U_{\Delta x}(x, \ell \Delta t) & :=U_{\Delta x}\left(\left(n+a_{\ell}\right) \Delta x, \ell \Delta t-0\right), \\
I_{\Delta x}(x, \ell \Delta t, \omega, \nu) & :=I_{\Delta x}\left(\left(n+a_{\ell}\right) \Delta x, \ell \Delta t-0, \omega, \nu\right),
\end{aligned}
$$

for $x \in[(n-1) \Delta x,(n+1) \Delta x], \quad n+\ell$ odd, $\omega \in[-1,1] . \nu \in \mathbb{R}_{+}$.

Then we solve in the same stroke, first the corresponding classical Riemann problem with initial data $U_{\Delta x}(x, \ell \Delta t)$, then the transport problem with initial data $I_{\Delta x}(x, \ell \Delta t, \omega, \nu)$, and finally the corresponding generalized problems with initial data $U_{\Delta x}(x, \ell \Delta t)$ and $I_{\Delta x}(x, \ell \Delta t, \omega, \nu)$ which allows to get $\left(I_{\Delta x}(x, t, \omega, \nu), U_{\Delta x}(x, t)\right)$ well defined in the strip $\{(x, t) \in \mathbb{R} \times[\ell \Delta t,(\ell+1) \Delta t]\}$. This process defines inductively $\left(I_{\Delta x}(x, t, \omega, \nu), U_{\Delta x}(x, t)\right)$ in the half-plane $\left\{(x, t) \in \mathbb{R} \times \mathbb{R}_{+}\right\}$.

Using the simplified notations $U_{\ell, n}:=U_{\Delta x}(\ell \Delta t, n \Delta x)$ for $\ell+n$ odd, and $I_{\ell, n}:=I_{\Delta x}(\ell \Delta t, n \Delta x, \omega, \nu)$ for $\ell+n$ odd, we have after (40) and (41) the value of the solution of the generalized Riemann (GR) problem

$$
U_{\ell, n}=V_{\ell, n}+\Delta \operatorname{tg}\left(\mathcal{I}_{\ell, n} ; V_{\ell, n}\right),
$$

and

$$
I_{\ell, n}=\mathcal{I}_{\ell, n}+c \Delta t \mathcal{S}\left(\mathcal{I}_{\ell, n} ; V_{\ell, n}\right)
$$

where $V_{\ell, n}$ is the value of the solution of the classical Riemann (CR) problem (38):

$$
C R U\left(n \Delta x,(\ell-1) \Delta t ; U_{\ell-1, n-1}, U_{\ell-1, n+1}\right),
$$


taken at the sample point $\left(\ell \Delta t,\left(n+a_{\ell}\right) \Delta x\right)$ i.e.

$$
V_{\ell, n}:=W_{C}\left(a_{\ell} \frac{\Delta x}{\Delta t}, n \Delta x,(\ell-1) \Delta t ; U_{\ell-1, n-1}, U_{\ell-1, n+1}\right),
$$

and $\mathcal{I}_{\ell, n}$ is the value of the solution of the problem

$$
C R I\left(n \Delta x,(\ell-1) \Delta t ; I_{\ell-1, n-1}, I_{\ell-1, n+1}\right),
$$

taken at the same sample point $\left(\ell \Delta t,\left(n+a_{\ell}\right) \Delta x\right)$ i.e.

$$
\mathcal{I}_{\ell, n}:=I_{C}\left(a_{\ell} \frac{\Delta x}{\Delta t},(\ell-1) \Delta t, n \Delta x ; I_{\ell-1, n-1}, I_{\ell-1, n+1}\right) .
$$

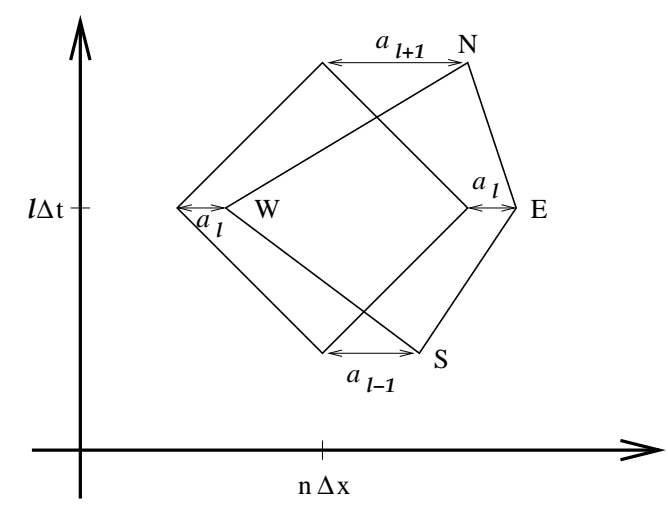

Figure 4: The diamond region $D_{\ell, n}$.

We define now [31] the diamond region $D_{\ell, n}$ as the convex hull of the points $S:=\left((\ell-1) \Delta t,\left(n+a_{\ell-1}\right) \Delta x\right)$, $W:=\left(\ell \Delta t,\left(n-1+a_{\ell}\right) \Delta x\right), E:=\left(\ell \Delta t,\left(n+1+a_{\ell}\right) \Delta x\right)$ and $N:=\left((\ell+1) \Delta t,\left(n+a_{\ell+1}\right) \Delta x\right)$, together with the "GR" values $U_{S}=U_{\ell-1, n}, U_{W}:=U_{\ell, n-1}, U_{E}:=U_{\ell, n+1}, U_{N}:=U_{\ell+1, n}$ and the "CR" values $V_{S}=V_{\ell-1, n}$, $V_{W}:=V_{\ell, n-1}, V_{E}:=V_{\ell, n+1}$ and $V_{N}:=V_{\ell+1, n}$.

An $i$-wave $\left(U_{i-1}(t), U_{i}(t)\right)$ in the approximate Riemann problem $U_{\Delta x}$ is defined as

$$
\left(U_{i-1}(t), U_{i}(t)\right)=\left(U_{i-1}, U_{i}\right)+(t-n \Delta t) g\left(\mathcal{I}_{\ell, n}, V_{\ell, n}\right)
$$

The strength of this $i$-wave is not clearly defined, so we set it equal to the strength of the wave $\left(U_{i-1}, U_{i}\right)$, as in $[12]$ :

$$
\varepsilon_{i}=\varepsilon_{i}\left(U_{i-1}, U_{i}\right)
$$

Let $J_{\ell}$ be a mesh curve located in the strip $\{(t, x) ; \ell \Delta t \leqslant t \leqslant(\ell+1) \Delta t\}$, and let $T V\left(U_{\Delta x}(t, x) ; J_{\ell}\right)$ (resp. $\left.T V\left(V_{\Delta x}(t, x) ; J_{\ell}\right)\right)$ the total variation of $U_{\Delta x}\left(\operatorname{resp} . V_{\Delta x}\right)$ on $J_{\ell}$. If an $i$-wave $\left(U_{i-1}(t), U_{i}(t)\right)$ of $U_{\Delta x}(t, x)$ originating in $(\ell \Delta t, n \Delta x)$ intersects $J_{\ell}$, then the corresponding classical $i$-wave $\left(U_{i-1}, U_{i}\right)$ of $V_{\Delta x}(t, x)$ also intersects $J_{\ell}$.

Following the ideas of Hong and LeFloch, we expect that the sum of the strength of the elementary waves in $U_{\Delta x}$ which cross a mesh $J$ can be considered as a measure equivalent to the total variation of $U_{\Delta x}$ : the radiative source $g$ will remain small at any time provided they actually are at $t=0$ and provided that the transport coefficients are smooth enough.

After formula (40)

$$
\left(U_{i-1}(t), U_{i}(t)\right)=\left(U_{i-1}, U_{i}\right)+\Delta t g\left(I_{\ell, n},\left(U_{i-1}, U_{i}\right)\right)
$$

Then

$$
\left|T V\left(U_{\Delta x}(t, x) ; J_{\ell}\right)-T V\left(V_{\Delta x}(t, x) ; J_{\ell}\right)\right| \leqslant \Delta t \sum_{n \in \mathbb{Z}} T V\left(g\left(\mathcal{I}_{\ell, n}, V_{\ell, n}\right) ; J_{\ell}\right)
$$


Let us assume that $(U, I) \in \mathcal{U}$ are such that $T V(U)$ and $\|T V(I)\|_{L^{1}\left(\mathbb{R}_{+} \times[-1,1]\right)}$ are small i.e.

$$
T V\{U\}+\|T V\{I\}\|_{L^{1}\left(\mathbb{R}_{+} \times[-1,1]\right)} \leqslant \varepsilon,
$$

for $\varepsilon>0$ small enough, and that hypotheses of Proposition 4 are satisfied.

Let us estimate the term $T V\left(g\left(\mathcal{I}_{\ell, n}, V_{\ell, n}\right) ; J_{\ell}\right)$ in the right-hand side of (73). As in Proposition 4, we must consider the two nonzero components of $g$.

We have then to estimate

and

$$
T V\left\{g_{2}\right\}:=T V\left\{\int_{\mathbb{R}_{+} \times[-1,1]} \mathcal{S}\left(I, U_{i}\right) d \omega d \nu\right\}
$$

$$
T V\left\{g_{3}\right\}:=T V\left\{\int_{\mathbb{R}_{+} \times[-1,1]} \omega \mathcal{S}\left(I, U_{i}\right) d \omega d \nu\right\} .
$$

As the transport coefficients do not depend on $\omega$, one gets first

$$
T V\left\{g_{2}\right\} \leqslant T V\left\{\int_{\mathbb{R}_{+}} \sigma_{a}\left(U_{i}, \nu\right) B\left(\nu, U_{i}\right) d \nu\right\}+T V\left\{\int_{\mathbb{R}_{+} \times[-1,1]} \sigma_{a}\left(U_{i}, \nu\right) I d \omega d \nu\right\},
$$

so using the identity $\int_{\mathbb{R}_{+}} B\left(\nu, U_{i}\right) d \nu=a \vartheta_{\ell, n}^{4}$ where $a$ is a pure positive constant, we have, using (21) and (22),

$$
\begin{aligned}
T V\left\{g_{2}\right\} \leqslant & a\left(4 \sup _{U \in \mathcal{U}}\left(\left|h_{a}(U)\right| \vartheta^{3}\right)+\sup _{U \in \mathcal{U}}\left(\left|\tilde{h}_{a}(U)\right| \vartheta^{4}\right)\right) \cdot T V\left\{U_{i}\right\} \overline{\sigma_{a}}(t) \\
& +\|T V\{I\}\|_{L^{1}\left(\mathbb{R}_{+} \times[-1,1]\right)} \sup _{U \in \mathcal{U}}\left|h_{a}(U)\right| \overline{\sigma_{a}}(t)+\|I\|_{L^{1}\left(\mathbb{R}_{+} \times[-1,1]\right)} \sup _{U \in \mathcal{U}}\left|\tilde{h}_{a}(U)\right| T V\left\{U_{i}\right\} \overline{\sigma_{a}}(t) .
\end{aligned}
$$

In the same stroke

$$
\begin{gathered}
T V\left\{g_{3}\right\}=T V\left\{\int_{\mathbb{R}_{+} \times[-1,1]}\left(\sigma_{a}\left(V_{\ell, n}, \nu\right)+\sigma_{s}\left(V_{\ell, n}, \nu\right)\right) \mathcal{I}_{\ell, n} d \omega d \nu\right\} \\
\leqslant\left(\sup _{U \in \mathcal{U}}\left|h_{a}(U)\right|\left\|T V\left\{\mathcal{I}_{\ell, n}\right\}\right\|_{L^{1}\left(\mathbb{R}_{+} \times[-1,1]\right)}+\sup _{U \in \mathcal{U}}\left|\tilde{h}_{a}(U)\right|\|I\|_{L^{1}\left(\mathbb{R}_{+} \times[-1,1]\right)} T V\left(V_{\ell, n}\right)\right) \overline{\sigma_{a}}(t) \\
+\left(\sup _{U \in \mathcal{U}}\left|h_{s}(U)\right|\left\|T V\left\{\mathcal{I}_{\ell, n}\right\}\right\|_{L^{1}\left(\mathbb{R}_{+} \times[-1,1]\right)}+\sup _{U \in \mathcal{U}}\left|\tilde{h}_{s}(U)\right|\|I\|_{L^{1}\left(\mathbb{R}_{+} \times[-1,1]\right)} T V\left(V_{\ell, n}\right)\right) \overline{\sigma_{s}}(t) .
\end{gathered}
$$

When $\left(U_{i-1}, U_{i}\right)$ is a rarefaction wave, the same computation shows that a similar estimate holds. Hence, summing over all the elementary waves crossing $J_{\ell}$, plugging into (73) and taking into account the CFL condition (67), we get

$$
\begin{gathered}
\left|T V\left(U_{\Delta x} ; J_{\ell}\right)-T V\left(V_{\Delta x} ; J_{\ell}\right)\right| \\
\leqslant O(\Delta t)\left(T V\left\{U_{\Delta x} ; J_{\ell}\right\}+T V\left\{\|I\|_{L^{1}\left(\mathbb{R}_{+} \times[-1,1]\right)}\right\}\right) .
\end{gathered}
$$

After (74) the right-hand side is small and we conclude that the total variations of $U_{\Delta x}$ and $V_{\Delta x}$ are equivalent on any mesh curve $J_{k}$.

We define the strength $\varepsilon_{i n}\left(D_{\ell, n}\right)$ of waves coming into $D_{\ell, n}$ for $\ell+n$ even by

$$
\varepsilon_{i n}\left(D_{\ell, n}\right)=\left|\varepsilon\left(V_{W}, U_{S} ;(\ell-1) \Delta t,(n-1) \Delta x\right)\right|+\left|\varepsilon\left(U_{S}, V_{E} ;(\ell-1) \Delta t,(n+1) \Delta x\right)\right|,
$$

and as well the strength $\varepsilon_{\text {out }}\left(D_{\ell, n}\right)$ of waves leaving $D_{\ell, n}$

$$
\varepsilon_{\text {out }}\left(D_{\ell, n}\right)=\left|\varepsilon\left(U_{W}, V_{N} ; \ell \Delta t, n \Delta x\right)\right|+\left|\varepsilon\left(V_{N}, U_{E} ; \ell \Delta t, n \Delta x\right)\right|=\left|\varepsilon\left(U_{W}, U_{E} ; \ell \Delta t, n \Delta x\right)\right| .
$$

We define analogous quantities for the radiative waves coming into $D_{\ell, n}$ for $\ell+n$ even

$$
\varepsilon_{r, i n}\left(D_{\ell, n}\right)=\int_{0}^{\infty} \int_{0}^{1}\left|\varepsilon_{r}\left(\mathcal{I}_{W}, I_{S} ;(\ell-1) \Delta t,(n-1) \Delta x\right)\right| d \omega d \nu
$$




$$
+\int_{0}^{\infty} \int_{-1}^{0}\left|\varepsilon_{r}\left(I_{S}, \mathcal{I}_{E} ;(\ell-1) \Delta t,(n+1) \Delta x\right)\right| d \omega d \nu,
$$

and as well the strength $\varepsilon_{r, o u t}\left(D_{\ell, n}\right)$ of waves leaving $D_{\ell, n}$

$$
\begin{aligned}
\varepsilon_{r, \text { out }}\left(D_{\ell, n}\right) & =\int_{0}^{\infty} \int_{-1}^{0}\left|\varepsilon_{r}\left(I_{W}, \mathcal{I}_{N} ; \ell \Delta t, n \Delta x\right)\right| d \omega d \nu \\
& +\int_{0}^{\infty} \int_{0}^{1}\left|\varepsilon_{r}\left(\mathcal{I}_{N}, I_{E} ; \ell \Delta t, n \Delta x\right)\right| d \omega d \nu .
\end{aligned}
$$

We also define the potential $\mathcal{P}\left(D_{\ell, n}\right)$ of interaction in the diamond $D_{\ell, n}$ for $\ell+n$ even, by

$$
\mathcal{P}\left(D_{\ell, n}\right):=D\left(\varepsilon\left(V_{W}, U_{S},(\ell-1) \Delta t,(n-1) \Delta x\right), \varepsilon\left(U_{S}, V_{E},(\ell-1) \Delta t,(n+1) \Delta x\right)\right),
$$

where $D(\cdot, \cdot)$ is defined by $(51)$.

Given a mesh curve $J$, one can split the waves crossing $J$ into two groups: the "left-incoming" waves (or type I waves) $\left(V_{\ell, n-1}, U_{\ell-1, n}\right)$, crossing the $W S$-type segments of $J$, and the "right-outgoing" waves (or type II waves) $\left(U_{\ell-1, n}, V_{\ell, n+1}\right)$, crossing the $S E$-type segments of $J$. Then we define the linear functional

$$
\begin{aligned}
& \mathcal{L}(J):=\sum_{\text {type } I \text { waves }}\left|\varepsilon\left(V_{\ell, n-1}, U_{\ell-1, n} ;(\ell-1) \Delta t,(n-1) \Delta x\right)\right| \\
& +\sum_{\text {type II waves }}\left|\varepsilon\left(U_{\ell-1, n}, V_{\ell, n+1} ;(\ell-1) \Delta t,(n+1) \Delta x\right)\right| \\
& +\sum_{\text {type I rad. waves }} \int_{0}^{\infty} \int_{0}^{1}\left|\varepsilon_{r}\left(\mathcal{I}_{\ell, n-1}, I_{\ell-1, n} ;(\ell-1) \Delta t,(n-1) \Delta x\right)\right| d \omega d \nu \\
& +\sum_{\text {type II rad. waves }} \int_{0}^{\infty} \int_{-1}^{0}\left|\varepsilon_{r}\left(I_{\ell-1, n}, \mathcal{I}_{\ell, n-1} ;(\ell-1) \Delta t,(n+1) \Delta x\right)\right| d \omega d \nu .
\end{aligned}
$$

Next, applying Glimm's strategy [9] (see also [28]), we correct $\mathcal{L}(J)$ by a quadratic term

$$
\mathcal{Q}(J):=\sum_{(\alpha, \beta)} D(\alpha, \beta)
$$

and we consider the final Glimm's functional

$$
\mathcal{F}(J):=\mathcal{L}(J)+K \mathcal{Q}(J)
$$

where $K>0$.

Note that due to the linear character of $C R I$, one does not need any quadratic correction for the radiative part.

Our aim is now to prove that $\mathcal{F}(J)$ is uniformly bounded for any mesh curve $J$ provided that the constant $K$ is large enough. As $\mathcal{F}(J)$ is equivalent to the total variation norm in $B V(\mathbb{R})$, we will conclude that $T V\left(U_{\Delta x}\right) \leqslant$ $C T V\left(U_{0}\right)$ which implies the convergence of the scheme by using a compactness argument.

Proposition 5. Let $J_{1}$ be a mesh curve, $J_{2}$ an immediate successor of $J_{1}$ and $D_{\ell_{0}, n_{0}}$ the diamond region determined by $J_{1}$ and $J_{2}$. The Glimm's functionals $\mathcal{L}\left(J_{1,2}\right)$ and $\mathcal{Q}\left(J_{1,2}\right)$ satisfy

$$
\mathcal{L}\left(J_{1}\right)-\mathcal{L}\left(J_{2}\right)=O(1)\left\{\mathcal{P}\left(D_{\ell_{0}, n_{0}}\right)+\varepsilon_{i n}\left(D_{\ell_{0}, n_{0}}\right) \Delta t\left(\overline{\sigma_{a}}\left(\left(\ell_{0}+1\right) \Delta t\right)+\overline{\sigma_{s}}\left(\left(\ell_{0}+1\right) \Delta t\right)\right)\right\}
$$

and

$$
\begin{aligned}
\mathcal{Q}\left(J_{1}\right)-\mathcal{Q}\left(J_{2}\right)=-\mathcal{P}\left(D_{\ell_{0}, n_{0}}\right)+O(1) \mathcal{L}\left(J_{1}\right) \mathcal{P}\left(D_{\ell_{0}, n_{0}}\right) & \\
& +O(1) \mathcal{L}\left(J_{1}\right) \varepsilon_{i n}\left(D_{\ell_{0}, n_{0}}\right) \Delta t\left(\overline{\sigma_{a}}\left(\left(\ell_{0}+1\right) \Delta t\right)+\overline{\sigma_{s}}\left(\left(\ell_{0}+1\right) \Delta t\right)\right)
\end{aligned}
$$

where the constants $C_{j}$ are defined in Proposition 4. 
Proof: From the previous definitions (75), (76) and (79), we have

$$
\mathcal{L}\left(J_{2}\right)-\mathcal{L}\left(J_{1}\right)=\varepsilon_{\text {out }}\left(D_{\ell_{0}, n_{0}}\right)-\varepsilon_{\text {in }}\left(D_{\ell_{0}, n_{0}}\right)+\varepsilon_{r, \text { out }}\left(D_{\ell_{0}, n_{0}}\right)-\varepsilon_{r, \text { in }}\left(D_{\ell_{0}, n_{0}}\right) .
$$

Using estimates (61) and (62) in Proposition 4, with $x_{0}=n_{0} \Delta x, t_{0}=\left(\ell_{0}-1\right) \Delta t, U_{L}=V_{W}, U_{M}=U_{S}$, $U_{R}=V_{E}, \mu_{L}=U_{W}-V_{W}, \mu_{R}=U_{E}-V_{E}$, we find

$$
\varepsilon_{\text {out }}\left(D_{\ell_{0}, n_{0}}\right)=\varepsilon_{\text {in }}\left(D_{\ell_{0}, n_{0}}\right)+O(1) \mathcal{P}\left(D_{\ell_{0}, n_{0}}\right)+O(1) \varepsilon_{\text {in }}\left(D_{\ell_{0}, n_{0}}\right) \Delta t\left(\overline{\sigma_{a}}\left(\left(\ell_{0}+1\right) \Delta t\right)+\overline{\sigma_{s}}\left(\left(\ell_{0}+1\right) \Delta t\right)\right),
$$

which gives (82).

As hydrodynamical and radiative elementary waves do not interact (83) directly follows from Proposition 4

In the spirit of [12] we have

Lemma 2. Let $\ell_{0}$ be a positive integer and suppose that $J_{1}$ (resp. $J_{2}$ ) is a mesh curve such that all the mesh points on $J_{1}$ (resp. $J_{2}$ ) belong either to the line $t=\left(\ell_{0}-1\right) \Delta t$ or to the line $t=\ell_{0} \Delta t$ (resp. the line $t=\ell_{0} \Delta t$ or to the line $\left.t=\left(\ell_{0}+1\right) \Delta t\right)$.

Suppose there exists a positive constant $M>0$ sufficiently small such that

$$
\mathcal{L}\left(J_{1}\right) \leqslant M \text {. }
$$

Then provided that the constant $K$ in (81) is large enough, the following estimate holds

$$
\mathcal{F}\left(J_{2}\right) \leqslant \mathcal{F}\left(J_{1}\right)+O(1) \Delta t\left(\overline{\sigma_{a}}\left(\left(\ell_{0}+1\right) \Delta t\right)+\overline{\sigma_{s}}\left(\left(\ell_{0}+1\right) \Delta t\right)\right) \sum_{n_{0} \in \mathbb{Z}} \varepsilon_{i n}\left(D_{\ell_{0}, n_{0}}\right)
$$

where $O(1)$ depends on $M, K$.

Proof: Fixing $n_{0} \in \mathbb{Z}$, we multiply (83) by $K$ and add it to (82). We obtain

$$
\begin{aligned}
\mathcal{F}\left(J_{2}\right)-\mathcal{F}\left(J_{1}\right)= & -K \sum_{n_{0} \in \mathbb{Z}} \mathcal{P}\left(D_{\ell_{0}, n_{0}}\right)+O(1)\left[1+K \mathcal{L}\left(J_{1}\right)\right] \sum_{n_{0} \in \mathbb{Z}} \mathcal{P}\left(D_{\ell_{0}, n_{0}}\right) \\
& +O(1)\left[1+K \mathcal{L}\left(J_{1}\right)\right] \sum_{n_{0} \in \mathbb{Z}}\left(\varepsilon_{i n}\left(D_{\ell_{0}, n_{0}}\right) \Delta t\left(\overline{\sigma_{a}}\left(\left(\ell_{0}+1\right) \Delta t\right)+\overline{\sigma_{s}}\left(\left(\ell_{0}+1\right) \Delta t\right)\right)\right) .
\end{aligned}
$$

Since $\sum_{n_{0} \in \mathbb{Z}} \mathcal{P}\left(D_{\ell_{0}, n_{0}}\right)=\mathcal{Q}\left(J_{1}\right)$, this implies

$$
\begin{aligned}
\mathcal{F}\left(J_{2}\right)-\mathcal{F}\left(J_{1}\right)= & -K \mathcal{Q}\left(J_{1}\right)+O(1)\left[1+K \mathcal{L}\left(J_{1}\right)\right] \mathcal{Q}\left(J_{1}\right) \\
& +O(1) \Delta t\left(\sum_{n_{0} \in \mathbb{Z}} \varepsilon_{i n}\left(D_{\ell_{0}, n_{0}}\right)\right)\left(\overline{\sigma_{a}}\left(\left(\ell_{0}+1\right) \Delta t\right)+\overline{\sigma_{s}}\left(\left(\ell_{0}+1\right) \Delta t\right)\right) \\
= & \mathcal{Q}\left(J_{1}\right)\left[K\left(O(1) \mathcal{L}\left(J_{1}\right)-1\right)+O(1)\right] \\
& +O(1) \Delta t\left(\sum_{n_{0} \in \mathbb{Z}} \varepsilon_{i n}\left(D_{\ell_{0}, n_{0}}\right)\right)\left(\overline{\sigma_{a}}\left(\left(\ell_{0}+1\right) \Delta t\right)+\overline{\sigma_{s}}\left(\left(\ell_{0}+1\right) \Delta t\right)\right) \\
\leqslant & \mathcal{Q}\left(J_{1}\right)[K(O(1) M-1)+O(1)] \\
& +O(1) \Delta t\left(\sum_{n_{0} \in \mathbb{Z}} \varepsilon_{i n}\left(D_{\ell_{0}, n_{0}}\right)\right)\left(\overline{\sigma_{a}}\left(\left(\ell_{0}+1\right) \Delta t\right)+\overline{\sigma_{s}}\left(\left(\ell_{0}+1\right) \Delta t\right)\right) .
\end{aligned}
$$

Choosing $M>0$ sufficiently small and $K>0$ sufficiently large, the first term of the right-hand side is negative, so (85) is proved.

Theorem 1. Let $\left(U_{\infty}, I_{\infty}\right) \in \mathcal{U}$ be a constant state, such that $I_{\infty}(\nu, \omega)=B\left(\nu, \vartheta_{\infty}\right)$, the temperature $\vartheta_{\infty}$ corresponding to $U_{\infty}$. Assume that the initial data $\left(U_{0}, I_{0}\right) \in \mathcal{U}$ are such that

$$
\left\|U_{0}-U_{\infty}\right\|_{L^{\infty}(\mathbb{R})},\left\|I_{0}-I_{\infty}\right\|_{L_{x}^{\infty}\left(\mathbb{R}, L_{\omega, \nu}^{1}\left([-1,1] \times \mathbb{R}_{+}\right)\right)},
$$

$T V\left(U_{0}\right), T V\left(\left\|I_{0}\right\|_{L^{\infty}\left([-1,1] \times \mathbb{R}_{+}\right)}\right)$are sufficiently small. 
Assume also that the following norms

$$
\int_{0}^{\infty} \overline{\sigma_{a}}(t) d t, \quad \int_{0}^{\infty} \overline{\sigma_{s}}(t) d t
$$

are sufficiently small. Then, the approximate solution $\left(U_{\Delta x}, I_{\Delta x}\right)$ are bounded uniformly in $L^{\infty}\left(\mathbb{R}^{+} \times \mathbb{R}\right)$ and in $B V\left(\mathbb{R}^{+} \times \mathbb{R}\right)$ :

$$
\begin{gathered}
\left\|U_{\Delta x}-U_{\infty}\right\|_{L^{\infty}([0, T] \times \mathbb{R})}+\left\|I_{\Delta x}-I_{\infty}\right\|_{L^{\infty}([0, T] \times \mathbb{R}), L^{1}\left([-1,1] \times \mathbb{R}^{+}\right)} \\
\leqslant O(1)\left(\left\|U_{0}-U_{\infty}\right\|_{L^{\infty}(\mathbb{R})}+\left\|I_{0}-I_{\infty}\right\|_{L_{x}^{\infty}\left(\mathbb{R}, L_{\omega, \nu}^{1}\left([-1,1] \times \mathbb{R}_{+}\right)\right)}+C\right) \\
T V\left(U_{\Delta x}\right)+\left\|T V\left(I_{\Delta x}\right)\right\|_{L_{\omega, \nu}^{1}\left([-1,1] \times \mathbb{R}_{+}\right)} \leqslant O(1)\left(T V\left(U_{0}\right)+\left\|T V\left(I_{0}\right)\right\|_{L_{\omega, \nu}^{1}\left([-1,1] \times \mathbb{R}_{+}\right)}+C\right)
\end{gathered}
$$

where

$$
C=\int_{0}^{\infty}\left(\overline{\sigma_{a}}(t)+\overline{\sigma_{s}}(t)\right) d t
$$

and $M$ is described in (84). Furthermore, $\left(U_{\Delta x}, I_{\Delta x}\right)$ is Lipschitz continuous in time, that is, for any $t_{1}, t_{2} \in$ $[0, T]$

$$
\begin{aligned}
\int_{\mathbb{R}}\left|U_{\Delta x}\left(t_{1}, x\right)-U_{\Delta x}\left(t_{2}, x\right)\right| d x+\left\|\int_{\mathbb{R}}\left|I_{\Delta x}\left(t_{1}, x\right)-I_{\Delta x}\left(t_{2}, x\right)\right| d x\right\|_{L_{\omega, \nu}^{1}\left([-1,1] \times \mathbb{R}_{+}\right)} \\
\quad \leqslant O(1)\left(\left|t_{1}-t_{2}\right|+\Delta t\right)\left(T V\left(U_{0}\right)+\left\|T V\left(I_{0}\right)\right\|_{L_{\omega, \nu}^{1}\left([-1,1] \times \mathbb{R}_{+}\right)}+C\right) .
\end{aligned}
$$

Proof: We first prove that condition (84) holds under the assumptions (86) and (87) by induction on $\ell_{0}$. For this purpose, we denote by $J_{\ell_{0}}$ a mesh curve which satifies the hypotheses of Lemma 2 , that is, any point of $J_{\ell_{0}}$ belongs either to the line $t=\left(\ell_{0}-1\right) \Delta t$ or to the line $t=\ell_{0} \Delta t$. For $\ell_{0}=0$, we clearly have

$$
\mathcal{F}\left(J_{0}\right) \leqslant O(1)\left(T V\left(U_{0}\right)+\left\|T V\left(I_{0}\right)\right\|_{L_{\omega, \nu}^{1}\left([-1,1] \times \mathbf{R}^{+}\right)}+K\left[T V\left(U_{0}\right)+\left\|T V\left(I_{0}\right)\right\|_{L_{\omega, \nu}^{1}\left([-1,1] \times \mathbf{R}^{+}\right)}\right]^{2}\right),
$$

where the term $O(1)$ depends only on the coefficients of the system. Hence, if $T V\left(U_{0}\right)+\left\|T V\left(I_{0}\right)\right\|_{L_{\omega, \nu}^{1}\left([-1,1] \times \mathbf{R}^{+}\right)}$ is sufficiently small, then $\mathcal{F}\left(J_{0}\right) \leqslant M$ hence $\mathcal{L}\left(J_{0}\right) \leqslant M$, where $M$ is defined in Lemma 2 .

Next, we assume that

$$
\forall \ell \leqslant \ell_{0}-1, \quad \mathcal{L}\left(J_{\ell}\right) \leqslant M
$$

Applying Lemma 2, we infer

$$
\mathcal{F}\left(J_{\ell_{0}}\right) \leqslant \mathcal{F}\left(J_{\ell_{0}-1}\right)+O(1) \Delta t \sum_{n_{0} \in \mathbb{Z}}\left(\overline{\sigma_{a}}\left(\left(\ell_{0}+1\right) \Delta t\right)+\overline{\sigma_{s}}\left(\left(\ell_{0}+1\right) \Delta t\right)\right) \varepsilon_{\mathrm{in}}\left(D_{\ell_{0}-1, n_{0}}\right) .
$$

Repeating this argument for each $\ell$ and summing from $\ell=0$ to $\ell=\ell_{0}-1$, we find

$$
\mathcal{F}\left(J_{\ell_{0}}\right) \leqslant \mathcal{F}\left(J_{0}\right)+O(1) \Delta t \sum_{n_{0} \in \mathbb{Z}} \sum_{\ell=0}^{\ell_{0}-1}\left(\overline{\sigma_{a}}((\ell+1) \Delta t)+\overline{\sigma_{s}}((\ell+1) \Delta t)\right) \varepsilon_{\text {in }}\left(D_{\ell, n_{0}}\right) .
$$

Then, we point out that

$$
\sum_{n_{0} \in \mathbb{Z}} \varepsilon_{\text {in }}\left(D_{\ell, n_{0}}\right)=\mathcal{L}\left(J_{\ell}\right)
$$

hence

$$
\mathcal{F}\left(J_{\ell_{0}}\right) \leqslant \mathcal{F}\left(J_{0}\right)+O(1) \Delta t \sum_{\ell=0}^{\ell_{0}-1}\left(\mathcal{L}\left(J_{\ell}\right)\left(\overline{\sigma_{a}}((\ell+1) \Delta t)+\overline{\sigma_{s}}((\ell+1) \Delta t)\right)\right) .
$$

Recalling (92) and (93), this implies

$$
\begin{array}{r}
\mathcal{F}\left(J_{\ell_{0}}\right) \leqslant O(1)\left(1+K T V\left(U_{0}\right)+\left\|T V\left(I_{0}\right)\right\|_{L_{\omega, \nu}^{1}\left([-1,1] \times \mathbf{R}^{+}\right)}\right) T V\left(U_{0}\right)+\left\|T V\left(I_{0}\right)\right\|_{L_{\omega, \nu}^{1}\left([-1,1] \times \mathbf{R}^{+}\right)} \\
+O(1) M \Delta t \sum_{\ell=0}^{\ell_{0}-1}\left(\overline{\sigma_{a}}((\ell+1) \Delta t)+\overline{\sigma_{s}}((\ell+1) \Delta t)\right) .
\end{array}
$$


Applying (90), this gives

$$
\mathcal{F}\left(J_{\ell_{0}}\right) \leqslant O(1)\left[\left(1+K T V\left(U_{0}\right)+\left\|T V\left(I_{0}\right)\right\|_{L_{\omega, \nu}^{1}\left([-1,1] \times \mathbf{R}^{+}\right)}\right) T V\left(U_{0}\right)+\left\|T V\left(I_{0}\right)\right\|_{L_{\omega, \nu}^{1}\left([-1,1] \times \mathbf{R}^{+}\right)}+C M\right] .
$$

Hence,

$$
\mathcal{L}\left(J_{\ell_{0}}\right) \leqslant O(1)\left[\left(1+K T V\left(U_{0}\right)+\left\|T V\left(I_{0}\right)\right\|_{L_{\omega, \nu}^{1}\left([-1,1] \times \mathbf{R}^{+}\right)}\right) T V\left(U_{0}\right)+\left\|T V\left(I_{0}\right)\right\|_{L_{\omega, \nu}^{1}\left([-1,1] \times \mathbf{R}^{+}\right)}+C M\right],
$$

where the term $O(1)$ is independent of $C, M, K$ and $T V\left(U_{0}\right)+\left\|T V\left(I_{0}\right)\right\|_{L_{\omega, \nu}^{1}\left([-1,1] \times \mathbf{R}^{+}\right)}$. Hence, we can choose $T V\left(U_{0}\right)+\left\|T V\left(I_{0}\right)\right\|_{L_{\omega, \nu}^{1}\left([-1,1] \times \mathbf{R}^{+}\right)}$small enough to have $O(1)\left(1+K T V\left(U_{0}\right)+\left\|T V\left(I_{0}\right)\right\|_{L_{\omega, \nu}^{1}\left([-1,1] \times \mathbf{R}^{+}\right)}\right) T V\left(U_{0}\right)+$ $\left\|T V\left(I_{0}\right)\right\|_{L_{\omega, \nu}^{1}\left([-1,1] \times \mathbf{R}^{+}\right)} \leqslant M / 2$, and $O(1) C M \leqslant M / 2$. Thus,

$$
\mathcal{L}\left(J_{\ell_{0}}\right) \leqslant M \text {. }
$$

This ends the induction proof.

We thus know that the sequence $\mathcal{L}\left(J_{\ell_{0}}\right)$ is bounded independently of $\ell_{0}$ and of $\Delta t$. Hence, since the functional $\mathcal{L}\left(J_{\ell_{0}}\right)$ is equivalent to the total variation of $U_{\Delta x}$, we deduce that the total variation of $U_{\Delta x}(t, x)$ is bounded independently of $t$ and $\Delta x$. this proves (89). The proof of (88) and (91) is dealt with exactly as in [9].

We are now in position to prove our main result:

Theorem 2. Under the hypotheses of Theorem 1, if the sequence $\left(a_{\ell}\right)_{\ell \geqslant 0}$ is equidistributed, the sequence $\left(U_{\Delta x}, I_{\Delta x}\right)$ defined by (69)-(70)-(71)-(72) converges in $L_{\text {loc }}^{1}$, up to extraction of a subsequence, to a function $(U, I)=(U(x, t), I(x, t))$ which is a solution of $(2)$, such that $U$ is an entropy solution of the first three equations of $(2)$.

Proof: Let $\left(U_{\Delta x}, I_{\Delta x}\right)$ be the solution defined by the generalized Glimm scheme (69)-(70)-(71)-(72). Applying Theorem 1 and Helly's theorem, there exists a subsequence of $\left(U_{\Delta x}, I_{\Delta x}\right)$ converging to $(U, I) \in L_{\text {loc }}^{1}$, which is in $B V$

Let $\theta$ be a test function in $\mathcal{D}\left(\mathbb{R}^{+} \times \mathbb{R}\right)$, and define

$$
R\left(U_{\Delta x}, I_{\Delta x}, \theta\right)=\int_{\mathbb{R}^{+}} \int_{\mathbb{R}}\left[U_{\Delta x} \partial_{t} \theta+f\left(U_{\Delta x}\right) \partial_{x} \theta+g\left(U_{\Delta x}, I_{\Delta x}\right) \theta\right] d x d t+\int_{\mathbb{R}} U_{\Delta x}(0, x) \theta(0, x) d x .
$$

The fact that $(U, I)$ is a weak solution of $(2)$ is exactly equivalent to $R(U, I, \theta)=0$ for any smooth $\theta$. The dominated convergence theorem implies that

$$
\lim _{\Delta x \rightarrow 0}\left|R\left(U_{\Delta x}, I_{\Delta x}, \theta\right)-R(U, I, \theta)\right|=0 .
$$

Hence, we are left with proving that

$$
\lim _{\Delta x \rightarrow 0} R\left(U_{\Delta x}, I_{\Delta x}, \theta\right)=0 .
$$

We split the integral defining $R\left(U_{\Delta x}, I_{\Delta x}, \theta\right)$ into a sum of integrals over the sets $Q_{\ell_{0}, n_{0}}=\left[\ell_{0} \Delta t,\left(\ell_{0}+1\right) \Delta t\right] \times$ $\left[\left(n_{0}-1\right) \Delta x,\left(n_{0}+1\right) \Delta x\right]$, for $\ell_{0} \in \mathbb{N}$ and $n_{0}+\ell_{0} \in 2 \mathbb{Z}$ :

$$
R\left(U_{\Delta x}, I_{\Delta x}, \theta\right)=\sum_{\ell_{0}=0}^{\infty} \sum_{n_{0}+\ell_{0} \in 2 \mathbb{Z}} \int_{\ell_{0} \Delta t}^{\left(\ell_{0}+1\right) \Delta t} \int_{\left(n_{0}-1\right) \Delta x}^{\left(n_{0}+1\right) \Delta x} U_{\Delta x} \partial_{t} \theta+f\left(U_{\Delta x}\right) \partial_{x} \theta+g\left(U_{\Delta x}, I_{\Delta x}\right) \theta .
$$


Applying Proposition 2, we thus have

$$
\begin{aligned}
R\left(U_{\Delta x}, I_{\Delta x}, \theta\right)= & O(1) \sum_{\ell_{0}=0}^{\infty} \sum_{n_{0}+\ell_{0} \in 2 \mathbb{Z}} O(1)\left(\Delta t^{2}+\Delta x^{2}\right)\left(\Delta x+\Delta t+\left|U_{\ell_{0}, n_{0}+1}-U_{\ell_{0}, n_{0}-1}\right|\right)\|\theta\|_{C^{1}\left(Q_{\ell_{0}, n_{0}}\right)} \\
& +\sum_{\ell_{0}=0}^{\infty} \sum_{n_{0}+\ell_{0} \in 2 \mathbb{Z}}\left(\int_{\left(n_{0}-1\right) \Delta x}^{\left(n_{0}+1\right) \Delta x} U_{\Delta x}\left(\left(\ell_{0}+1\right) \Delta t-, x\right) \theta\left(\left(\ell_{0}+1\right) \Delta t, x\right) d x\right. \\
& \left.-\int_{\left(n_{0}-1\right) \Delta x}^{\left(n_{0}+1\right) \Delta x} U_{\Delta x}\left(\ell_{0} \Delta t+, x\right) \theta\left(\ell_{0} \Delta t+, x\right) d x\right)+\int_{\mathbb{R}} U_{\Delta x}(0, x) \theta(0, x) d x \\
& +\sum_{\ell_{0}=0}^{\infty} \sum_{n_{0}+\ell_{0} \in 2 \mathbb{Z}}\left(\int_{\ell_{0} \Delta t}^{\left(\ell_{0}+1\right) \Delta t} f\left(t,\left(n_{0}+1\right) \Delta x, U_{\Delta x}\left(t,\left(n_{0}+1\right) \Delta x-\right)\right) d t\right. \\
& \left.-\int_{\ell_{0} \Delta t}^{\left(\ell_{0}+1\right) \Delta t} f\left(t,\left(n_{0}-1\right) \Delta x, U_{\Delta x}\left(t,\left(n_{0}-1\right) \Delta x+\right)\right) d t\right) .
\end{aligned}
$$

We denote by $R_{1}$ the first line of the right-hand side, by $R_{2}$ the second and third lines, and by $R_{3}$ the fourth and fifth lines: $R\left(U_{\Delta x}, I_{\Delta x}, \theta\right)=R_{1}+R_{2}+R_{3}$. We deal with each term separately: for $R_{1}$, we apply (89), and find that

$$
\left|R_{1}\right| \leqslant C \Delta x+C \sum_{\ell_{0}=0}^{\infty} \sum_{n_{0}+\ell_{0} \in 2 \mathbb{Z}}\left(\Delta x^{2}+\Delta t^{2}\right)\left(1+T V\left(U_{0}\right)+\left\|T V\left(I_{0}\right)\right\|_{L_{\omega, \nu}^{1}\left([-1,1] \times \mathbf{R}^{+}\right)}\right)\|\theta\|_{C^{1}\left(Q_{\ell_{0}, n_{0}}\right)},
$$

hence

$$
\left|R_{1}\right| \leqslant C \Delta x
$$

Turning to $R_{2}$, we have

$$
R_{2}=-\sum_{\ell_{0}=0}^{\infty} \sum_{n_{0}+\ell_{0} \in 2 \mathbb{Z}} \int_{\left(n_{0}-1\right) \Delta x}^{\left(n_{0}+1\right) \Delta x}\left[U_{\Delta x}\right]\left(\ell_{0} \Delta t, x\right) \theta\left(\ell_{0} \Delta t, x\right) d x
$$

where [.] denotes the jump of a function: $\left[U_{\Delta x}\right]\left(\ell_{0} \Delta t, x\right)=U_{\Delta x}\left(\ell_{0} \Delta t+, x\right)-U_{\Delta x}\left(\ell_{0} \Delta t-, x\right)$. We apply a result by Liu [22], which implies that, since the sequence $\left(a_{n}\right)_{n \in \mathbb{Z}}$ is equidistributed,

$$
\lim _{\Delta x \rightarrow 0}\left(\sum_{\ell_{0}=0}^{\infty} \sum_{n_{0}+\ell_{0} \in 2 \mathbb{Z}} \int_{\left(n_{0}-1\right) \Delta x}^{\left(n_{0}+1\right) \Delta x}\left[U_{\Delta x}\right]\left(\ell_{0} \Delta t, x\right) \theta\left(\ell_{0} \Delta t, x\right) d x\right)=0 .
$$

Hence,

$$
\lim _{\Delta x \rightarrow 0} R_{2}=0
$$

Turning to $R_{3}$, we have, according to the definition of $U_{\Delta x}$ and since $g$ is smooth,

$$
\begin{aligned}
\left|R_{3}\right| & \leqslant C \sum_{\ell_{0}=0}^{\infty} \sum_{n_{0}+\ell_{0} \in 2 \mathbb{Z}} \int_{\ell_{0} \Delta t}^{\left(\ell_{0}+1\right) \Delta t}\left|U_{\Delta x}\left(t,\left(n_{0}+1\right) \Delta t+\right)-U_{\Delta x}\left(t,\left(n_{0}-1\right) \Delta t-\right)\right|\|\theta\|_{C^{1}\left(Q_{\ell_{0}, n_{0}}\right)} d t \\
& \leqslant C \sum_{\ell_{0}=0}^{\infty} \sum_{n_{0}+\ell_{0} \in 2 \mathbb{Z}} \int_{\ell_{0} \Delta t}^{\left(\ell_{0}+1\right) \Delta t} t\left|g\left(\ell_{0} \Delta t,\left(n_{0}+2\right) \Delta x, U_{\ell_{0}, n_{0}+1}\right)-g\left(\ell_{0} \Delta t, n_{0} \Delta x, U_{\ell_{0}, n_{0}+1}\right)\right| d t\|\theta\|_{C^{1}\left(Q_{\ell_{0}, n_{0}}\right)} \\
& \leqslant C \sum_{\ell_{0}=0}^{\infty} \sum_{n_{0}+\ell_{0} \in 2 \mathbb{Z}} \int_{\ell_{0} \Delta t}^{\left(\ell_{0}+1\right) \Delta t} t \Delta x d t\|\theta\|_{C^{1}\left(Q_{\ell_{0}, n_{0}}\right)} .
\end{aligned}
$$

Hence,

$$
\left|R_{3}\right| \leqslant C \Delta x
$$

We then collect (95), (96), (97), and find that (94) holds. That is to say, $U$ is a weak solution of (2). As far as the radiative part is concerned, the preceding proof applies in a simpler way, since we do not use any Riemann problem for this part. 
It remains to prove that $(U, I)$ is an entropy solution, that is, for any entropy pair $(S, F)$ and any non-negative test function $\theta$, we have

$$
\int_{\mathbb{R}^{+}} \int_{\mathbb{R}} S(U) \partial_{t} \theta+F(U) \partial_{x} \theta+P(U) \theta+\int_{\mathbb{R}} S\left(U_{0}\right) \theta(0, x) d x \geqslant 0,
$$

with

$$
P=\partial_{U} S g(t, x, U)+\partial_{x} F(t, x, U) .
$$

The proof of this fact follows exactly the same line as that of (94), applying Proposition 2.

\section{Acknowledgements}

The authors are grateful to Prof. F. Golse for interesting discussions on the subject.

\section{References}

[1] A. Bourgeade, Ph. Le Floch, P.A. Raviart. An asymptotic expansion for the solution of the generalized Riemann problem. Part 2: application to the equations of gas dynamics. Ann. I.H.P. Analyse non linéaire, 6:437-480, 1989.

[2] A. Bressan. Hyperbolic systems of conservation laws. Oxford University Press, 2000.

[3] C. Buet, B. Després. Asymptotic analysis of fluid models for the coupling of radiation and hydrodynamics. Journal of Qantitative Spectroscopy and Radiative Transfer, 85:385-418, 2004.

[4] S. Chandrasekhar. Radiative transfer. Dover Publications, Inc., New York, 1960.

[5] C. Dafermos. Hyperbolic conservation laws in continuum physics. Springer Verlag, Berlin, Heidelberg, New York, 2000.

[6] C. Dafermos, L. Hsiao. Hyperbolic systems of balance laws with inhomogeneity and dissipation. Indiana Univ. Math. Journal, 31:471-491, 1982.

[7] B. Dubroca, J.-L. Feugeas. Etude théorique et numérique d'une hiérarchie de modèles aux moments pour le transfert radiatif. C. R. Acad. Sci. Paris, 329 Série I:915-920, 1999.

[8] G. Frosali, C. V. M. van der Mee, S. L. Paveri-Fontana, Conditions for runaway phenomena in the kinetic theory of particle swarms. J. Math. Phys. 30, no. 5, 1177-1186, 1989.

[9] J. Glimm. Solutions in the large for nonlinear hyperbolic systems of conservation laws. Comm. Pure Appl. Math., 18:697-715, 1965.

[10] F. Golse, G. Allaire, Transport et diffusion. Lecture Notes, Ecole polytechnique, 2010.

[11] F. Golse, B. Perthame. Generalized solutions of the radiative transfer equations in a singular case. Commun. Math. Phys., 106:211-239, 1986.

[12] J.M. Hong, Ph. Le Floch. A version of the Glimm method based on generalized Riemann problems. Port. Math., 64:199-236, 2007.

[13] L. Hörmander. Lectures on nonlinear hyperbolic differential equations. Springer Verlag, Berlin, Heidelberg, New York, 1997.

[14] L. Hsiao. Quasilinear hyperbolic systems and dissipative mechanisms. World Scientific, Singapore, New Jersey, London, Hong Kong, 1997.

[15] P. Jiang, D. Wang. Formation of singularities of solutions to the three-dimensional Euler-Boltzmann equations in radiation hydrodynamics. Preprint, March 11, 2009. 
[16] P. Jiang, D. Wang. Global weak solutions to the Euler-Boltzmann equations in radiation hydrodynamics. Preprint, June 27, 2009.

[17] Ph.G. Le Floch. Hyperbolic conservation of conservation laws. Birkhäuser Verlag, Basel, Boston, Berlin, 2002.

[18] Ph.G. Le Floch, T.P. Liu. Existence theory for nonlinear hyperbolic systems. Forum Math., 5:261-280, 1993.

[19] Ph.G. Le Floch, P.A. Raviart. An asymptotic expansion for the solution of the generalized Riemann problem. Part 1: general theory. Ann. I.H.P. Analyse non linéaire, 5:179-209, 1988.

[20] C. Lin. Mathematical analysis of radiative transfer models. PhD Thesis, 2007.

[21] C. Lin, J.-F. Coulombel, T. Goudon. Shock profiles for non-equilibrium radiating gases. Physica D, 218:83-94, 2006.

[22] T.-P. Liu. The deterministic version of the Glimm scheme. Commun. Math. Phys., 57:135-148, 1977.

[23] T.-P. Liu. Quasilinear hyperbolic systems. Commun. Math. Phys., 68:141-172, 1979.

[24] T.-P. Liu. Admissible solutions of hyperbolic conservation laws. Memoirs AMS, 30:N0. $240,1981$.

[25] R.B. Lowrie, J.E. Morel, J.A.. Hittinger. The coupling of radiation and hydrodynamics. The Astrophysical Journal, 521:432-450, 1999.

[26] D. Mihalas, B. Weibel-Mihalas. Foundations of radiation hydrodynamics. Oxford University Press, New York, 1984.

[27] G.C. Pomraning. Radiation hydrodynamics. Dover Publications, Inc., Mineola, New York, 2005.

[28] M. Schatzman. Continuous Glimm functionals and uniqueness of solutions of the Riemann problem. Indiana Univ. Math. J., 34:533-589, 1985.

[29] D. Serre. Systèmes de lois de conservation I. Diderot Editeur, Arts et Sciences, Paris, New-York, Amsterdam, 1996.

[30] R.G. Smith. The Riemann problem in gas dynamics. Transactions of the AMS, 249:1-50, 1979.

[31] J. Smoller. Shock waves and reaction-diffusion equations. Springer Verlag, Berlin, Heidelberg, New York, 1983.

[32] Ya.B. Zel'dovich, Yu.P. Raiser. Physics of shock waves and high-temperature hydrodynamic phenomena. Dover Publications, Inc., Mineola, New York, 2002.

[33] X. Zhong, S. Jiang. Local existence and finite-time blow-up in multidimensional radiation hydrodynamics. J. Math fluid mech., 9:543-564, 2007.

\author{
Bernard Ducomet \\ CEA, DAM, DIF \\ F-91297 Arpajon, France \\ E-mail: bernard.ducomet@cea.fr \\ Xavier Blanc \\ Laboratoire Jacques-Louis Lions \\ Université Paris Diderot \\ 5 rue Thomas Mann \\ 75205 Paris Cedex 13 \\ E-mail: blanc@ann.jussieu.fr
}

